2011-6

\title{
Compressibility Effects of Extended Formation Flight
}

Andrew Ning

Brigham Young University, aning@byu.edu

Ilan Kroo

Stanford University

Follow this and additional works at: https://scholarsarchive.byu.edu/facpub

Part of the Mechanical Engineering Commons

\section{Original Publication Citation}

Ning, A., and Kroo, I., "Compressibility Effects of Extended Formation Flight," 29th AIAA Applied Aerodynamics Conference, Honolulu, Hawaii, Jun. 2011. doi:10.2514/6.2011-3812

\section{BYU ScholarsArchive Citation}

Ning, Andrew and Kroo, Ilan, "Compressibility Effects of Extended Formation Flight" (2011). Faculty Publications. 1748.

https://scholarsarchive.byu.edu/facpub/1748

This Conference Paper is brought to you for free and open access by BYU ScholarsArchive. It has been accepted for inclusion in Faculty Publications by an authorized administrator of BYU ScholarsArchive. For more information, please contact ellen_amatangelo@byu.edu. 


\title{
Compressibility Effects of Extended Formation Flight
}

\author{
S. Andrew Ning* and Ilan Kroo ${ }^{\dagger}$ \\ Stanford University, Stanford, CA, 94305
}

\begin{abstract}
Aircraft flown in formations may realize significant reductions in induced drag by flying in regions of wake upwash. However, most transports fly at transonic speeds and compressibility effects in formation flight are not well understood. This study uses an Euler solver to analyze the inviscid aerodynamic forces and moments of transonic wing/body configurations flying in a 2-aircraft formation. We consider formations with large streamwise separation distances (10-50 wingspans) in an arrangement we term extended formation flight.

Compressibility-related drag penalties in formation flight may be eliminated by slowing 2-3\% below the nominal out-of-formation drag divergence Mach number, at fixed lift coefficient or fixed altitude. The latter option has the additional benefit that the aerodynamic performance of the formation improves slightly at higher lift coefficients. Optimal in-formation lift coefficients are not nearly as high as those estimated by incompressible analyses, but if not limited by engine performance, modest increases in altitude can yield further improvements in aerodynamic efficiency. Increasing the lateral separation of the aircraft can allow for slightly higher cruise speeds in exchange for higher induced drag. For the configurations examined here, a 1-2\% reduction in Mach number combined with a lateral spacing increase of $5 \%$ span achieves a total formation drag savings of about $10 \%$.
\end{abstract}

\section{Nomenclature}

$\begin{array}{ll}R & \text { aspect ratio } \\ b & \text { wingspan } \\ b_{0} & \text { separation distance between vortex pair } \\ c & \text { chord } \\ C_{D} & \text { drag coefficient } \\ C_{D p} & \text { parasitic drag coefficient } \\ C_{L} & \text { lift coefficient } \\ C_{p} & \text { pressure coefficient } \\ f & \text { functional } \\ L / D & \text { lift-to-drag ratio } \\ M_{\infty} & \text { freestream Mach number } \\ N_{c} & \text { number of cells } \\ r & \text { radial position } \\ S_{r e f} & \text { reference wing area } \\ U_{\infty} & \text { freestream velocity } \\ V & \text { velocity } \\ V_{\theta} & \text { radial velocity } \\ V_{z} & \text { vertical component of velocity } \\ x & \text { streamwise position } \\ y & \text { lateral position } \\ y_{t i p} & \text { lateral position of wingtip relative to vortex center }\end{array}$

*Doctoral Candidate, Department of Aeronautics and Astronautics, aning@stanford.edu, AIAA Student Member.

${ }^{\dagger}$ Professor, Department of Aeronautics and Astronautics, AIAA Fellow. 
$z \quad$ vertical position

Symbols

$\Gamma_{0} \quad$ vortex circulation

$\lambda$ taper ratio

$\Lambda_{Q C} \quad$ wing quarter-chord sweep

$\nu \quad$ dynamic viscosity

$\omega \quad$ vorticity

\section{Introduction}

With the world fleet projected to approximately double in size over the next twenty years, ${ }^{1,2}$ much effort is being devoted to further increase the efficiency of air transportation. Formation flight is one area of interest for its potential to significantly reduce fuel consumption of long range flights. Drag reduction through formation flight is not a new concept, and has been demonstrated in simulations, experiments, and flight tests. ${ }^{3-8}$

While the benefits of close formation flight have been known for some time, our recent analyses suggest that significant drag savings can be realized even when the aircraft are separated streamwise by ten to fifty spans, in an arrangement we term extended formation flight. ${ }^{9}$ These extended formations allow the aircraft to fly at safer separation distances while still retaining much of the formation benefits. However, for aircraft that fly at transonic speeds, some of the benefits may be negated or unrealizable due to compressibility effects. The upwash from a lead aircraft's wake can cause a fairly high spanwise variation in induced angle of attack on the following aircraft (as high as approximately 6 degrees near the vortex core relative to far from the core). Transonic sections can have poor off-design performance at their design cruise speeds, and the modified load distribution may lead to shocks, flow separation, buffet, and increased drag.

There are several ways to reduce the compressibility penalties due to formation flying including alleviating the spanwise loading, redesigning the wing, flying further from the center of the vortex, and slowing down. In order to trim the aircraft in roll, some load redistribution is necessary. However, the level of load tailoring achievable may not be sufficient to prevent increases in compressibility drag. Redesigning the wing to account for multiple design conditions may be beneficial, but presents its own challenges and would not help existing aircraft. Flying further from the center of the vortex will reduce the variation in induced angles of attack, but will also reduce the induced drag savings. Finally, slowing down presents the most obvious solution, but longer flight times can negatively impact the economics of the aircraft's mission.

This study analyzes the inviscid aerodynamic performance of formations of aircraft flying at transonic speeds, separated streamwise by ten or more spans. These studies can be used to provide guidance for subsequent viscous solutions, studies using more detailed configurations, and experimental work. For simplicity, results focus on a two-aircraft formation of identical aircraft. Geometry changes are not considered in the scope of this study.

The paper is divided into three main sections. First, an overview of the methodology is given. Second, a comparison between two different wake development methods is presented. Finally, inviscid performance for a 2-aircraft formation with variation in Mach number, lateral position, and lift coefficient is evaluated.

\section{Methods}

The large streamwise separation distances involved in extended formation flight make high-fidelity simulations of the formation in a single domain impractical. Fortunately, because of the large distances, the trailing aircraft has negligible influence on the lead aircraft. This allows the problem to be decomposed into three separable phases which are solved sequentially: lead aircraft, wake propagation, and trailing aircraft. We explore two such approaches for computing the aerodynamic forces of a formation of aircraft as diagramed in Figure 1. The first approach starts with the lead aircraft evaluated in its own domain. The vorticity field on a plane one span behind the lead aircraft is extracted, and used to initialize a 2D Navier-Stokes solver. Past studies have shown that the wake development phase is very nearly two-dimensional for the distances of interest in this application. ${ }^{10}$ This solution is then propagated forward in time to five spans in front of the trailing aircraft. This distance is still far enough away that the influence of the trailing aircraft can be assumed to be negligible. In the final phase, the propagated velocity field is imposed as an upstream 
boundary condition on a domain containing the trailing aircraft.

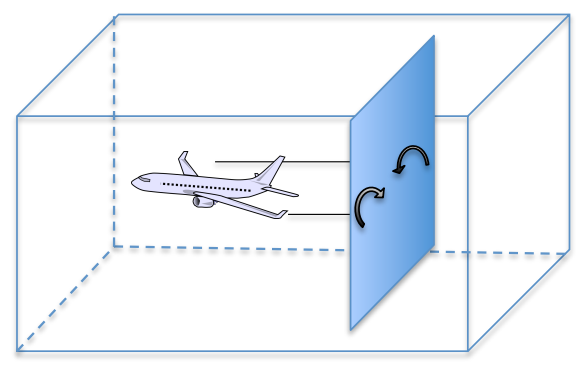

A1: AERO package (Euler) $\sim 450$ CPU hours

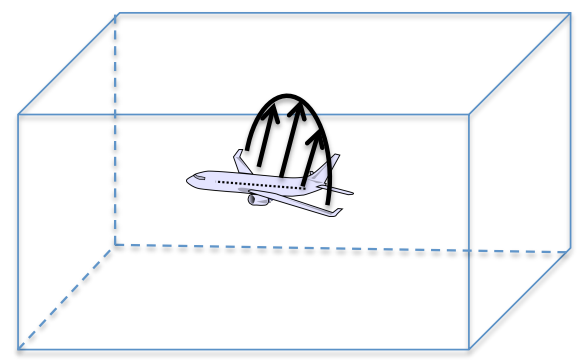

A2: AERO package (Euler) $\sim 150$ CPU hours

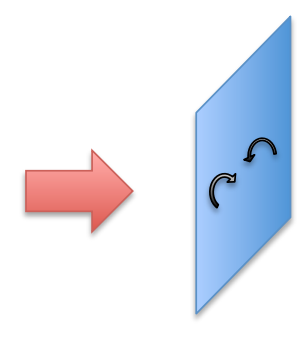

B1: VTEXE (Navier-Stokes) 1 hour / 10 spans
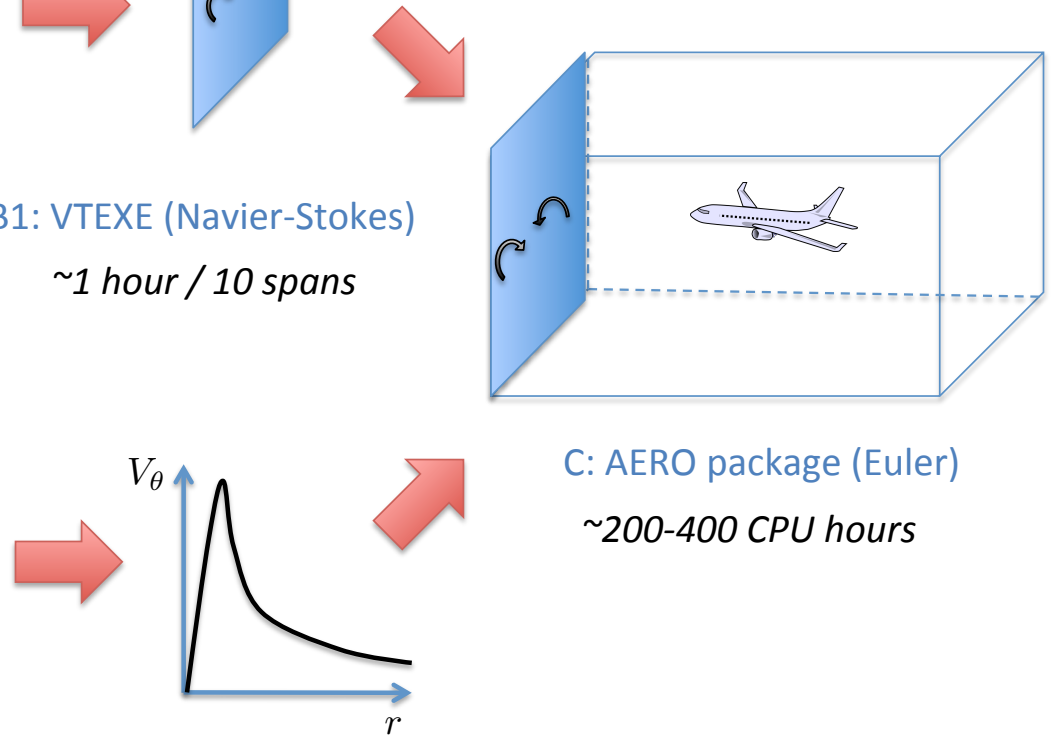

C: AERO package (Euler)

200-400 CPU hours

Figure 1. Overview of two different methods for resolving the aerodynamic forces for a formation of aircraft. First approach computes inviscid flow over lead aircraft, then extracts vorticity in a plane behind the aircraft (A1). Vorticity field is then evolved with a 2D Navier-Stokes solver (B1). Finally, resulting velocity field is imposed as a boundary condition on domain containing the trailing aircraft (C). Second approach computes inviscid flow over lead aircraft and extracts lift distribution (A2). A far-field conservation of vorticity method combined with empirical data is then used to "rollup" the wake (B2). The names of the various solvers, and some representative run-times are given for each stage (approximate timing for $1.6 \mathrm{GHz}$ Itanium 2 processors).

The second approach starts similarly with the lead aircraft in its own domain. However, instead of extracting vorticity, the lift distribution of the aircraft is resolved. This lift distribution is then "rolled up" using a far-field analysis method augmented with empirical data. Finally, the velocity field from the vortices is imposed as a boundary condition on the domain containing the trailing aircraft. More details on each phase of the methodology is discussed below.

\section{A. Phase A1: Vorticity Behind Lead Aircraft}

The objective of this analysis is to obtain the vorticity field on a plane one span behind the aircraft, orthogonal to the freestream direction. An Euler solution is desired for this analysis over lower-fidelity methods, so that the effects of a three-dimensional geometry on the vorticity distribution, as well as compressibility effects on the spanwise loading can be captured. A Navier-Stokes solution is deemed unnecessary as the early rollup behind an aircraft is essentially an inviscid process. ${ }^{11}$

The solver used in this analysis is NASA's AERO package, a Cartesian mesh Euler solver with adjoint driven mesh adaptation. ${ }^{12-14}$ The mesh is adaptively constructed by refining cells that contribute most to discretization errors in user-selected functionals. The functional used in all results unless stated otherwise is $C_{L}^{2} /\left(\pi R C_{\text {Dinviscid }}\right)$. This functional is similar to span efficiency, but uses the inviscid drag rather than the induced drag. Convergence in this functional allows for tight convergence in lift and drag simultaneously. This solver is especially advantageous for extended formation flight analyses as the wake must be propagated significant distances downstream, and the mesh adaptation strategy allows this to be done efficiently. 
The solution is performed in a frame which aligns the freestream velocity vector with the streamwise coordinate of the Cartesian mesh, with the geometry rotated to obtain the desired angle of attack. This is done so that vorticity can be extracted in a plane normal to the freestream direction. In order to obtain the vorticity distribution downstream of the aircraft, a second functional is added to drive the mesh adaptation. This functional is a "line sensor" which minimizes the error in computed pressure along a prescribed line. The line sensor is placed in a plane one span behind the aircraft essentially along the trace of the wing but descended by about $1 \%$ in span. Only a general placement of the sensor is needed, as the mesh adaptation will refine around the vortex as necessary in order to minimize error in pressure. An example of the mesh refinement in a plane one span behind a wing/body/nacelle geometry is shown in Figure 2 (with an inset view of the geometry).

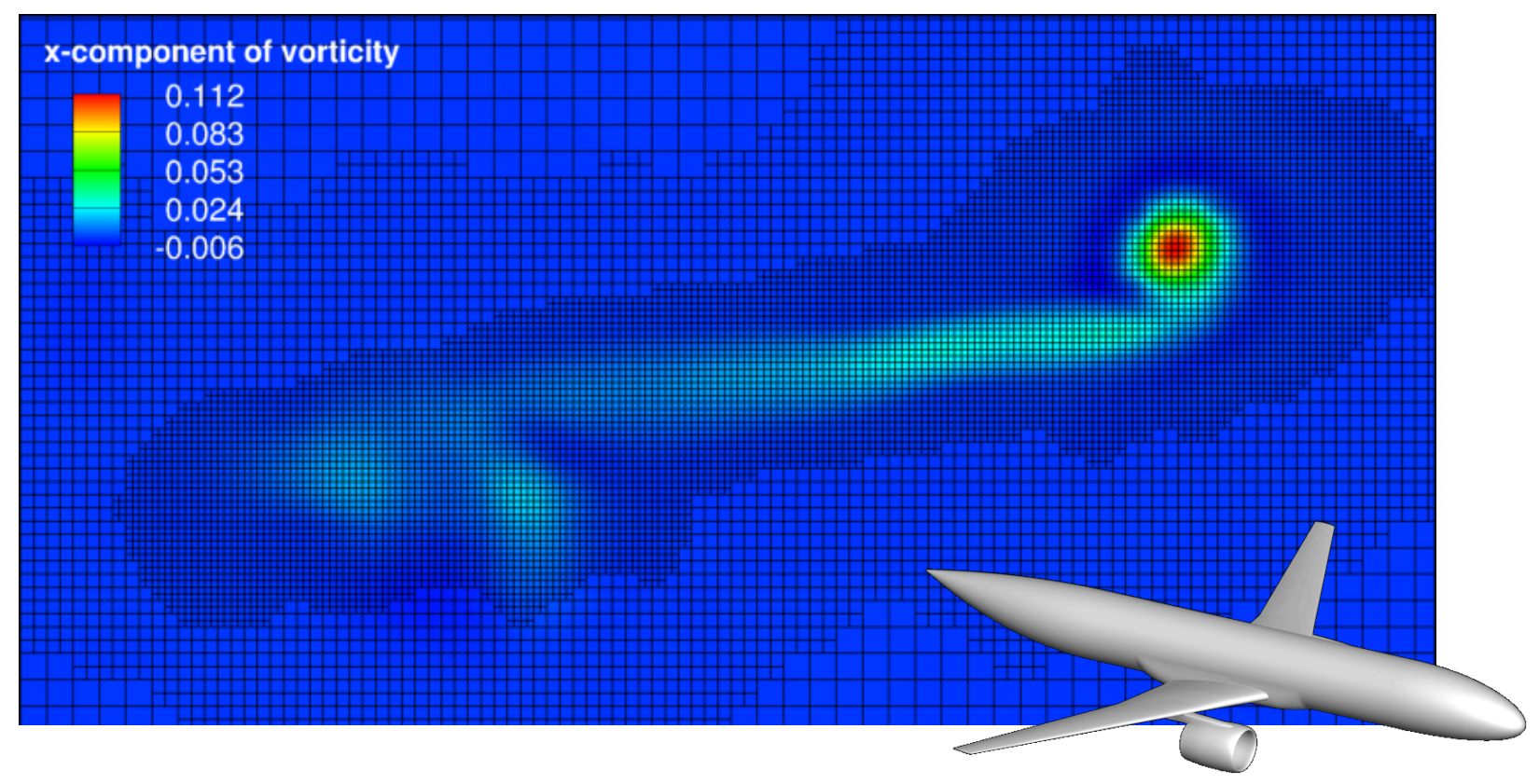

Figure 2. Contours of the component of vorticity in the freestream direction on a plane one span behind a wing/body/nacelle geometry. Mesh is automatically refined to capture the wake.

The domain boundaries are approximately 15 span lengths away from the aircraft in each direction, and a symmetry plane is used. As the velocities vary linearly across each cell volume, analytic gradients are used to compute a constant vorticity for each cell. The out-of-plane component of vorticity on the plane is then extracted for the next phase.

\section{B. Phase B1: Navier-Stokes Propagation of Wake Downstream}

As the wake is propagated downstream to distances of 10 to 50 spans, viscous effects can become important. However, as mentioned, the wake development process is still highly two-dimensional at these time scales, ${ }^{10}$ allowing the use of a two-dimensional solver to a good approximation. The three-dimensional nature of the wake, as well as the effects of turbulence, would be important to consider if larger aircraft separation distances were desired.

The solver used for this analysis is VTEXE, a Direct Numerical Simulation (DNS) code developed by Steve Rennich at Stanford University, that solves the incompressible vorticity equation

$$
\frac{\partial \boldsymbol{\omega}}{\partial t}+\nabla \times(\boldsymbol{\omega} \times \mathbf{V})=\nu \nabla^{2} \boldsymbol{\omega}
$$

using a psuedospectral approach. Boundary conditions are handled using the approach of Rennich and Lele. ${ }^{15}$ The method allows for vorticity that is spatially compact in two unbounded directions and is periodic in the third direction. 
These solutions use only one cell volume in the third direction, making it a 2D Navier-Stokes solver. The domain size is 1.6 span lengths in both directions, with a $512 \times 512$ uniform mesh. The domain is required to be square in this solver, and the size of 1.6 spans was chosen as the minimum size (with some padding) in which vorticity goes to zero at the bounds. Grid resolution studies were run on $128 \times 128,256 \times 256,512 \times 512$, and $1024 \times 1024$ meshes, and the $512 \times 512$ was found to be a appropriate choice for the domain size. A Reynolds number based on circulation of 10 million is used. After propagation, the downstream vorticity and velocity field is obtained for use in simulation of the trailing aircraft.

\section{Phase A2: Lift Distribution of Lead Wing}

The second approach uses a far-field wake rollup method that requires the lift distribution of the aircraft as input. The lift distribution could certainly be estimated by a panel method or even be prescribed, but an Euler analysis provides a more accurate lift distribution and captures compressibility effects on the spanwise loading. This phase involves only a straightforward flow solution for a single aircraft using the AERO package.

\section{Phase B2: Augmented Betz Wake Development}

In our previous work, ${ }^{9}$ we developed a methodology to estimate the wake development process using Betz's method for computing a far-field vorticity distribution, ${ }^{16}$ experimental data on viscous core size, ${ }^{17}$ and a wake decay model based on analytic/LES/experimental work of Holzäpfel. ${ }^{18}$ This method has the advantage that it is very fast to evaluate, but does not have the same fidelity as the Navier-Stokes solver.

This method uses a functional form for the radial velocity as seen in Figure 3. It includes a linear solidbody rotation section, a quadratic section containing the peak velocity at the core, a section defined by the Betz methodology ${ }^{16}$ which is defined piecewise linearly with logarithmic spacing to better define the rapid velocity drop near the core, and the far field solution for a vortex of given circulation strength. The velocity at any point in the domain is then given by the superposition of the velocities from all vortices defined on the boundary.

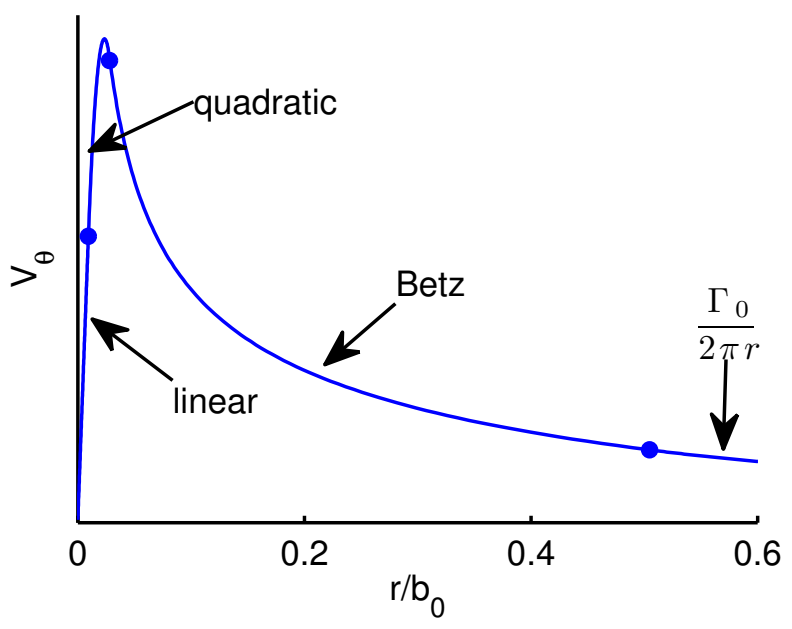

Figure 3. Functional form for the radial velocity from a vortex. There are four sections, a linear section, a quadratic section, a section defined by the Betz method, and a far-field vortex section.

This approach loses some accuracy as the real vorticity distribution is not perfectly radially symmetric, and does not follow this functional form exactly in a given direction. However, we do not need a perfectly accurate velocity field everywhere in the domain as long as it is accurate in the region where the trailing aircraft is flying. The impact of using this vortex model as opposed to the Navier-Stokes propagation method will be examined in more detail in a later section. 


\section{E. Phase C: Solving for Flow over Trailing Aircraft}

However it is generated, the velocity distribution from the lead aircraft's wake is now imposed as an upstream boundary condition for a second domain containing the trailing aircraft. It is desirable to keep the upstream boundary close to the trailing aircraft in order to reduce computational costs, but still far enough away that the trailing aircraft has had negligible impact on the wake development and that boundary effects are minimal. A boundary placement study concluded that a domain size of five spans in all directions was sufficient. Each cell volume is stretched streamwise with an aspect ratio of about 1.7. This is done in order to reduce the number of cells needed to bring in the influence of the wake from the boundary. Convergence studies found this to be a good balance between the need for higher spanwise resolution in the wake region versus higher streamwise resolution near the body (both areas need high vertical resolution). An example of the evolution of the mesh for the trailing aircraft can be seen in Figure 4 which shows a cut of the mesh in a plane just above the aircraft. In addition to the body refinement, the mesh refines along the path of wake development bringing its influence in from the upstream boundary.

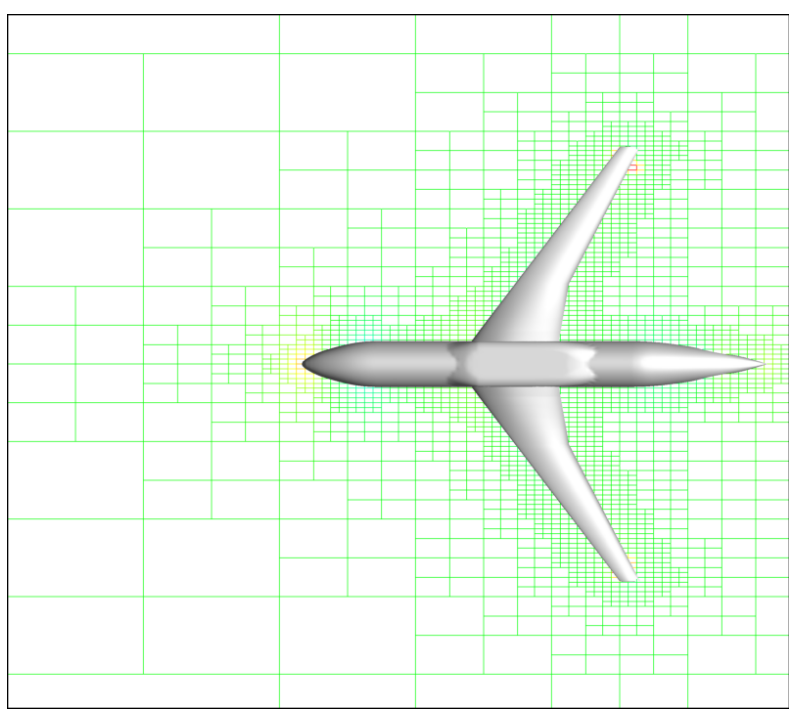

(a) initial mesh

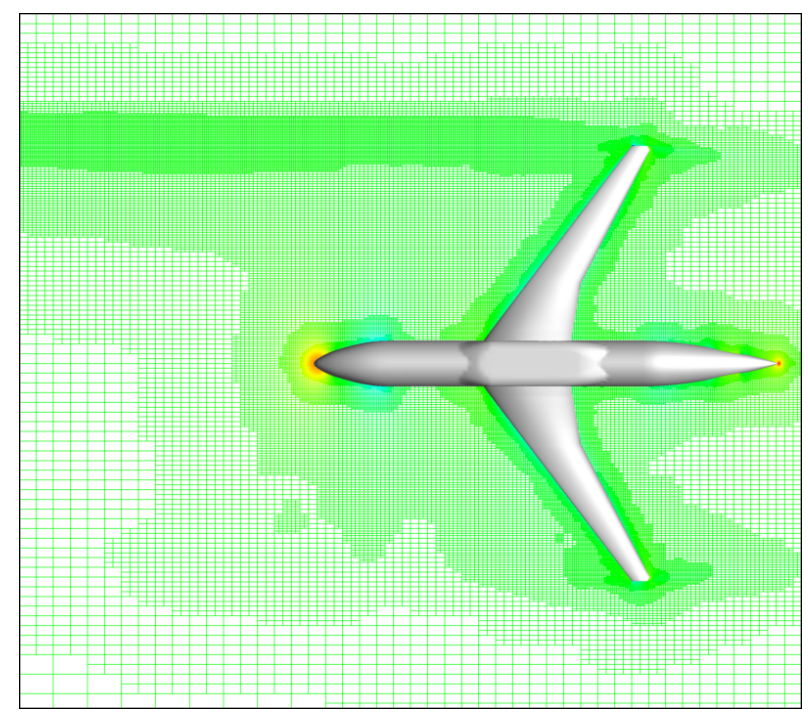

(b) final mesh

Figure 4. Cut plane passing near wingtip, with view looking up toward bottom of aircraft. Cells are colored by the local pressure. Evolution of mesh is shown, with final mesh showing refinement of vortex path from upstream boundary.

All cases for the trailing aircraft are run adaptively up to approximately 25-30 million cells. This is a reasonable size given the number of cases and amount of resources available. However, at these cell counts, not all cases reach complete convergence. Richardson extrapolation is used to estimate the functional based on the results of the last three intermediate solutions from the mesh adaptation strategy. In the asymptotic convergence regime the functional can be related to the cell size as follows

$$
f=f^{*}+m\left(\frac{1}{N_{c}^{1 / 3}}\right)^{p}
$$

where $p$, the order of convergence, has been shown to be 2 for this code. ${ }^{12,14}$ This approach has been shown to work well with the AERO package in previous studies. ${ }^{19}$ A typical convergence plot and extrapolated line is shown in Figure 5.

In many cases a target lift coefficient or position of the vortex is sought. For the lead aircraft this is straightforward, as the AERO package can periodically adjust the freestream angle of attack in order to obtained a desired lift coefficient. However this method cannot be used for the trailing aircraft domain, as the freestream angle of attack must remain at zero in order for the wake to propagate in the freestream direction. This requires that the geometry be rotated instead to achieve the desired lift coefficient. However, the proper angle of attack to achieve a given lift coefficient in-formation is not known a priori. In order to avoid extra CFD iterations, a vortex lattice method combined with our simple wake model ${ }^{9}$ is used to aid 


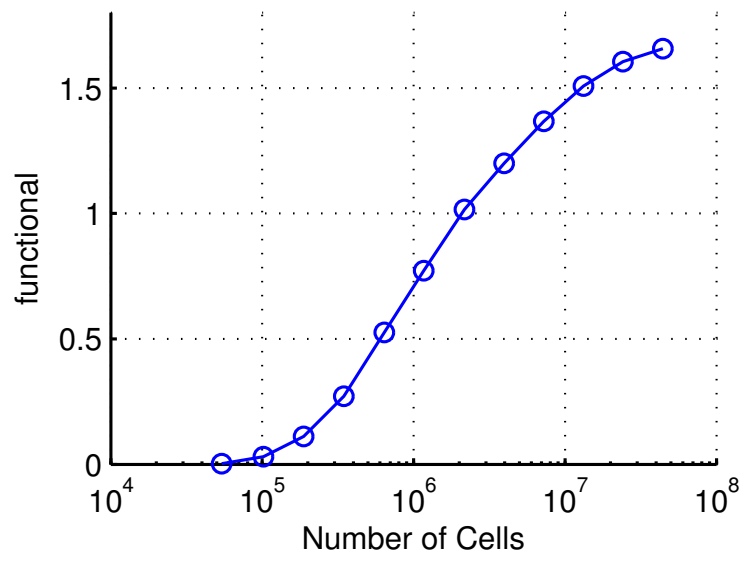

(a) Convergence in functional $\left(C_{L}^{2} /\left(\pi R C_{D i n v i s c i d}\right)\right)$
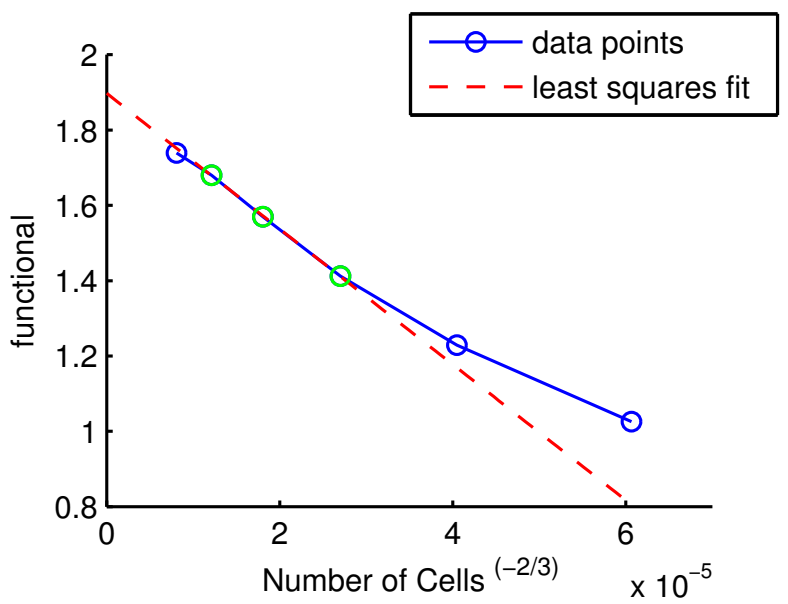

(b) Richardson extrapolation with least squares fit through green data points. Last point not included because most results terminate one iteration earlier

Figure 5. A typical convergence plot for a transonic wing/body in-formation. This particular case was run out to $44 \mathrm{M}$ cells, though most results are terminated at $25 \mathrm{M}$ cells with continuum values estimated from Richardson extrapolation.

in the estimation of the proper angle of attack. While this low-fidelity method does not provide an accurate direct estimate of the angle of attack, it does provide a good estimate for the relative change in angle of attack required by moving in and out of formation. Since the out-of-formation angle of attack is already solved for in the lead aircraft domain, a good estimate for the angle of attack required for the trailing aircraft can be obtained. For all results presented here, this method was sufficiently accurate to reach the desired lift coefficient within approximately $1 \%$ without the need to re-run the Euler solver a second time.

Similarly, for vortex positioning studies, an estimate is needed of the vortex descent distance from the boundary of the domain to the wing. The same low-fidelity method was used to estimate relative descent distances, and was also found to be accurate within $1 \%$ without iteration. A typical example, showing the location of the vortex relative to the wingtip is shown in Figure 6 . This case targeted positioning the wingtip at the center of the vortex, and the actual position is very close to the estimated position. The figure shows contours of the component of vorticity in the freestream direction.

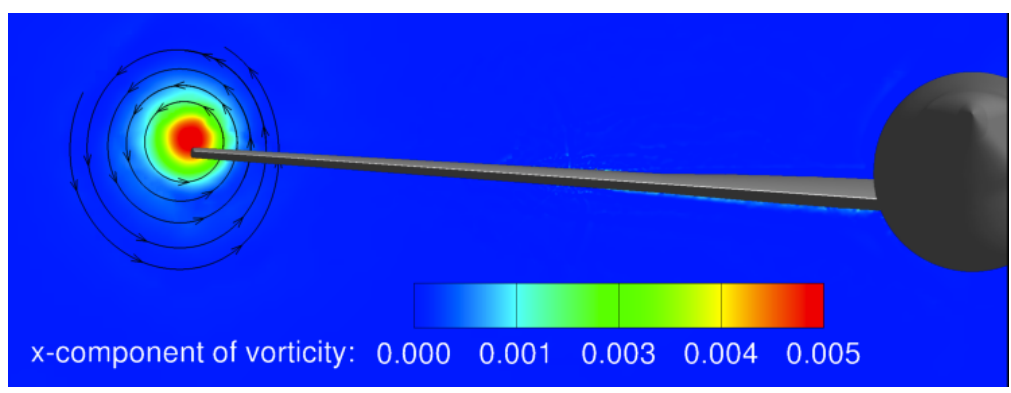

Figure 6. Contours of the component of vorticity in the freestream direction are shown on a cut through the wing for a transonic wing/body. A few confined streamlines near the wingtip are also shown.

\section{Wake Development Method}

This section explores in more detail methods for estimating wake velocities at a downstream location. The two methods are compared on three different geometries: a low speed wing, a transonic wing/body, and a transonic wing/body/nacelle. 


\section{A. Comparison Between the Two Different Approaches}

The first geometry is a simple untwisted aspect ratio 8 wing with NACA 0012 sections (Figure 7(a)). The wing is flown at Mach 0.5 with a lift coefficient of 0.55 . Figure 7(b) shows the vorticity distribution extracted from the Euler solution one span downstream of the wing, and the propagated Navier-Stokes solution twenty spans downstream of the wing. This is a symmetric solution with only the vorticity behind the right semi-span shown. The vorticity has rolled up into a tighter spiral and has descended under the influence of the other vortex.

The vertical velocity along a cut across the center of the vortex is shown as a function of spanwise distance in Figure 7(c). This velocity profile is compared to the results of the Augmented Betz method. The figure also shows how the two different methods compare in terms of drag reduction on a trailing aircraft in-formation. The induced drag fraction is the total induced drag of all aircraft in-formation relative to all aircraft out-of-formation. For the comparative purposes of this analysis, drag is evaluated using the faster incompressible analysis only. Results have been run out to 50 spans, but only the 20 span distance is shown as differences from 10-50 spans are relatively minor for both sets of analyses.

The same set of analyses are shown for a transonic wing/body geometry (Figure 8), and for a transonic wing/body/nacelle geometry (Figure 9). The two approaches show generally good agreement (particularly outboard of the vortex), although the Augmented Betz method tends to under-predict drag savings. This is not too surprising, as the parameters used were intentionally chosen to be conservative in our previous work. ${ }^{9}$ The chosen core size was at the higher end of the experimental data. ${ }^{17}$ Additionally, the decay model used ${ }^{18}$ includes a reduction in circulation even at time zero. If a core size near the average of the experimental data is used, and the viscous decay parameter is instead defined relative to its initial value, then the Augmented Betz results become almost indistinguishable from the CFD results for the first two geometries. Although arguably better accuracy may be obtained by adjusting these viscous decay parameters, they were not modified in these results in order to allow for a more direct comparison to our previous incompressible results. ${ }^{9}$

The final geometry (wing/body/nacelle) differs in that the conservative parameters for Augmented Betz show better agreement with the CFD results. This is due to the secondary vortex generated behind that aircraft with a nacelle and pylon. The Betz method assumes all of the vorticity rolls up into a single vortex and thus over-predicts the vortex strength for cases with multiple shed vortices. Although Extended Betz methods have been developed to try to predict cases with multiple vortices, ${ }^{20,21}$ they rely mainly on heuristics and were not explored here.

For all formation results discussed subsequently in this paper the second approach (the AERO package lift dist + Augmented Betz) will be used. This method certainly provides a speed advantage over the Euler/Navier-Stokes method, however that was not the only reason for its use. The other significant advantage is that the Augmented Betz vortex model is piecewise analytic, and can easily be evaluated at any level of grid fineness with minimal storage requirements. The difference in predicted drag savings between the two models differs only by a few percent. Negative $y_{t i p}$ values, corresponding to an overlap of the wingtip with the vortex, are of little practical interest for formation flight. For positive $y_{t i p}$ spacings the agreement between the two methods is even better. Thus, the more expensive simulations were deemed unnecessary for this analysis. For more detailed configurations, particularly those with deflected controls surfaces, and for larger formations where there is multiple wake interaction, the Navier-Stokes simulations may be necessary. 


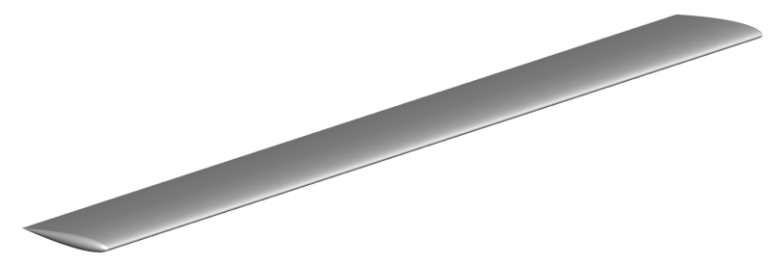

(a) Low speed wing geometry
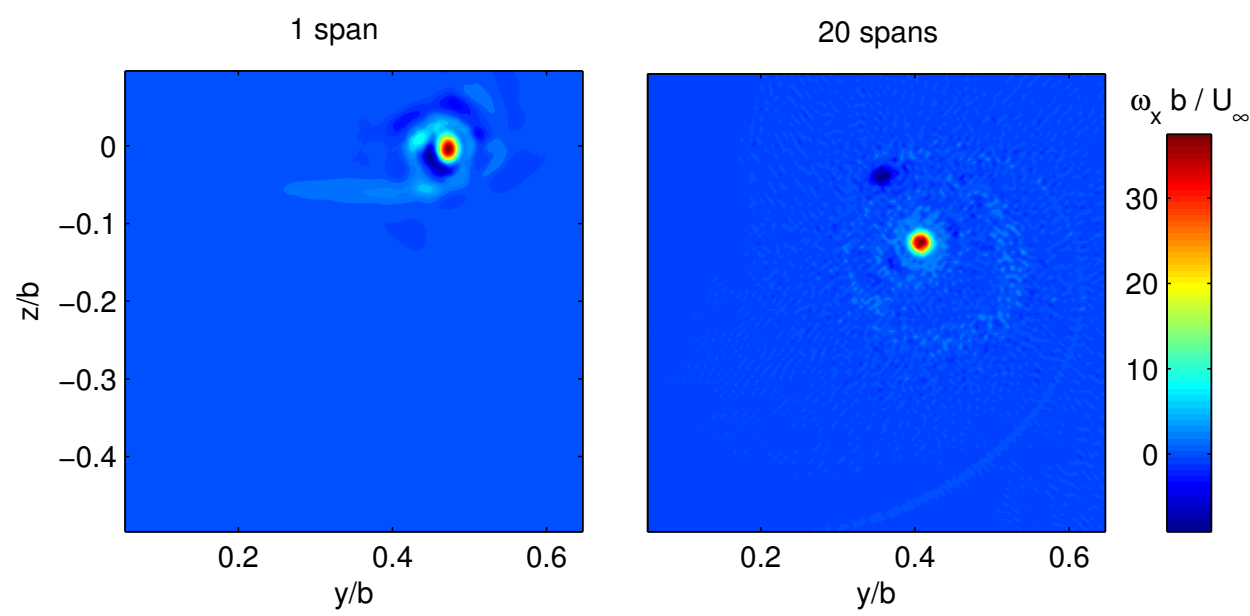

(b) Normalized x-component of vorticity 1 and 20 spans downstream (only right semi-span shown, Navier-Stokes propagation for second condition)
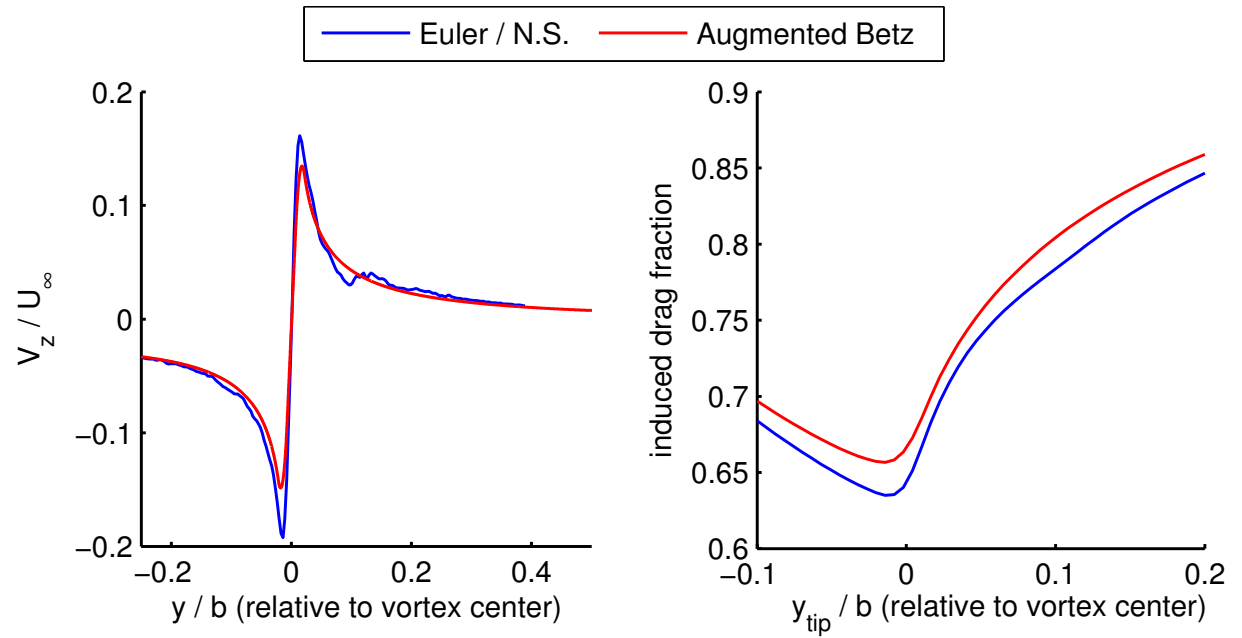

(c) Difference between high and low fidelity methods (20 spans downstream). Left figure shows upwash as a function of spanwise position on a cut through center of vortex. Right figure shows difference in estimated induced drag fraction for a trailing aircraft.

Figure 7. Wake development behind a low speed wing $\left(M_{\infty}=0.5, C_{L}=0.55\right)$. 


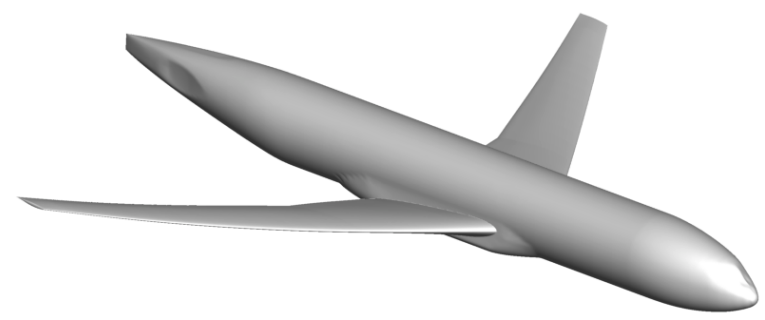

(a) Transonic wing/body geometry
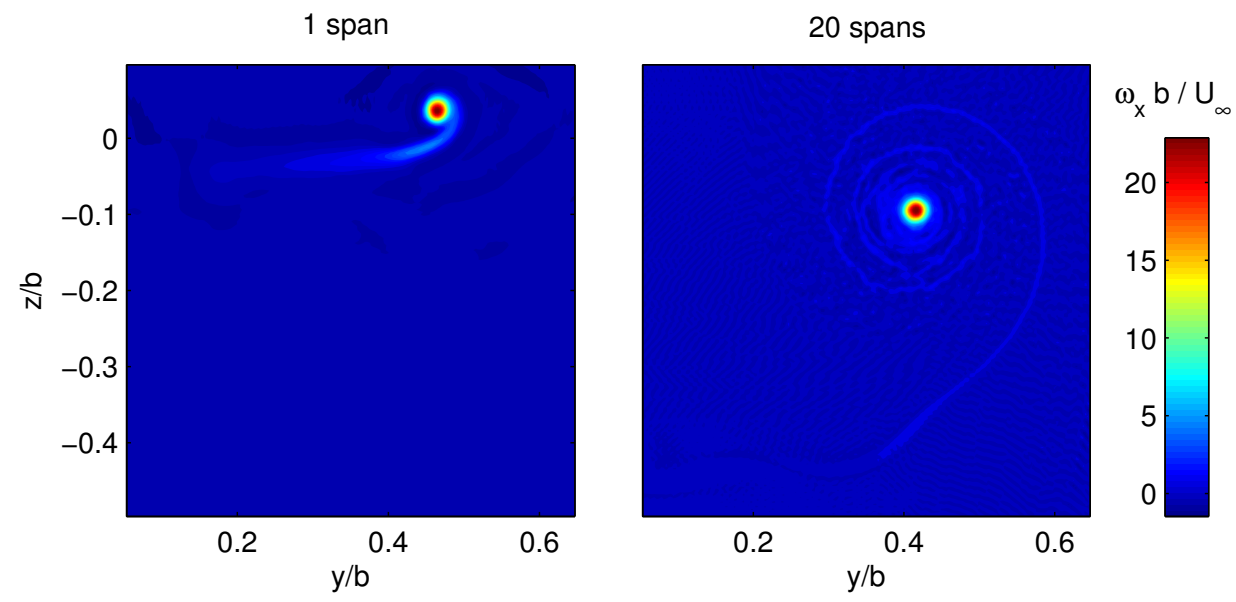

(b) Normalized x-component of vorticity 1 and 20 spans downstream (only right semi-span shown, Navier-Stokes propagation for second condition)

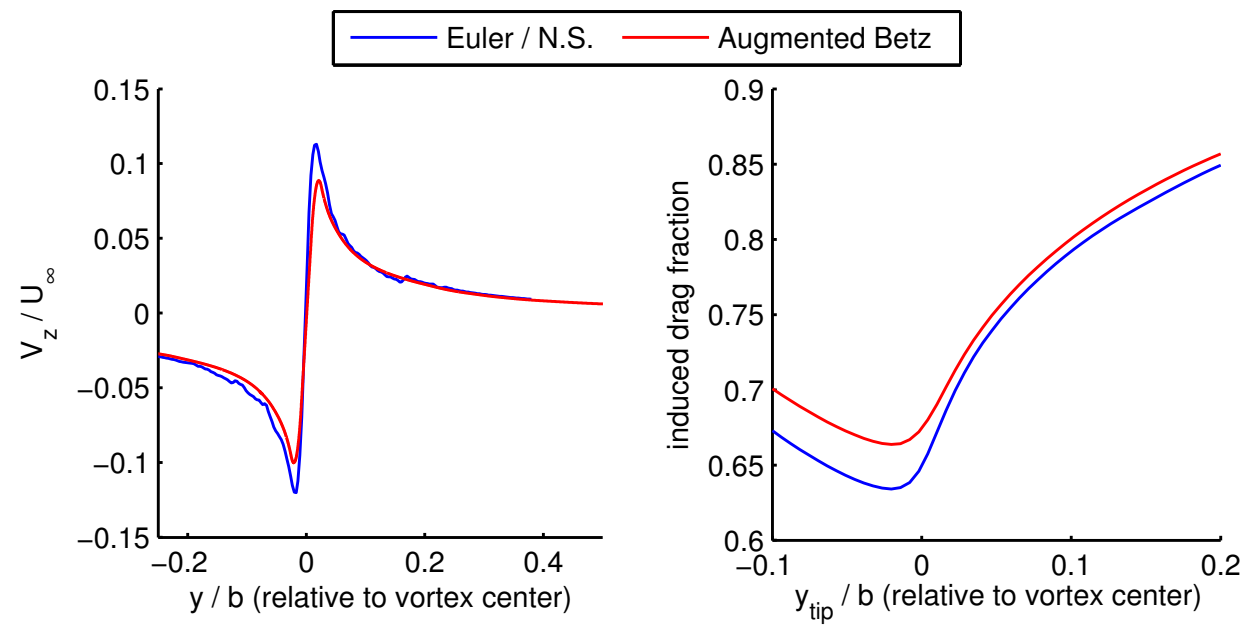

(c) Difference between high and low fidelity methods (20 spans downstream). Left figure shows upwash as a function of spanwise position on a cut through center of vortex. Right figure shows difference in estimated induced drag fraction for a trailing aircraft.

Figure 8. Wake development behind a transonic wing $/ \operatorname{body}\left(M_{\infty}=0.83, C_{L}=0.5\right)$. 


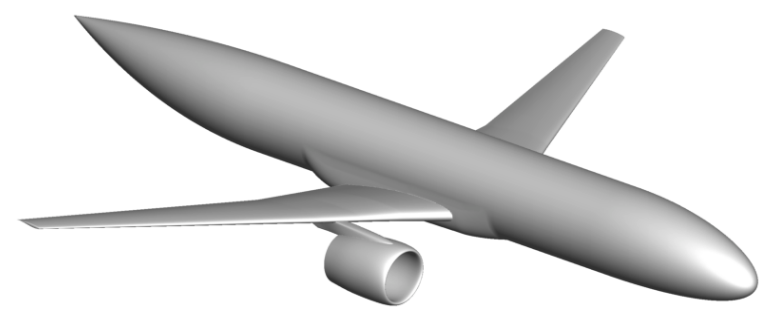

(a) Transonic wing/body/nacelle geometry
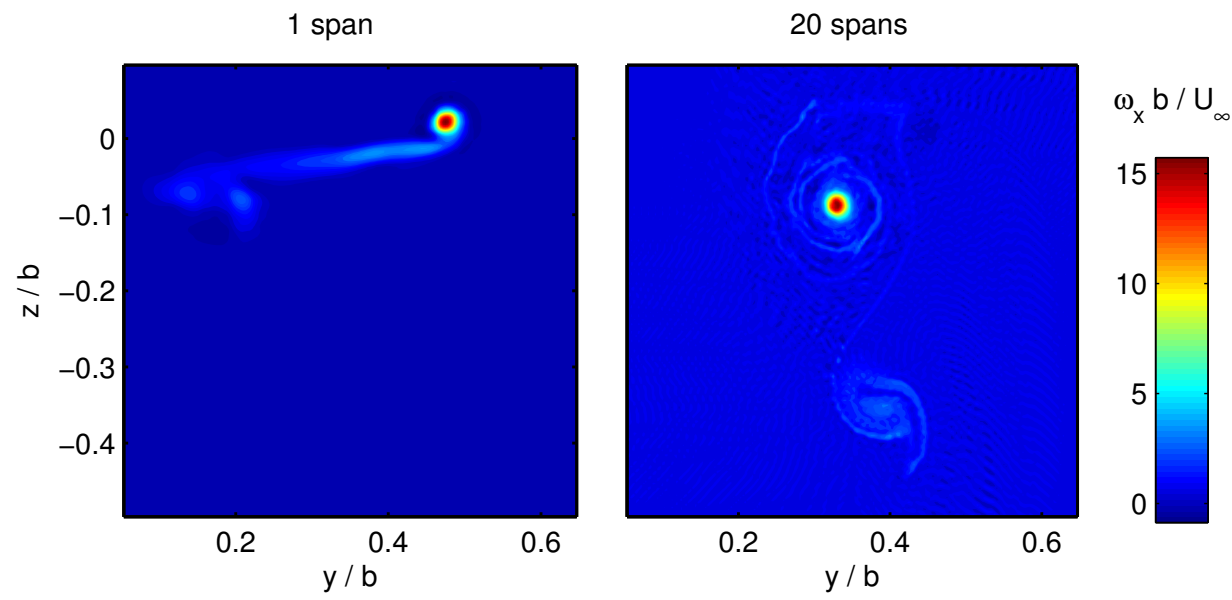

(b) Normalized x-component of vorticity 1 and 20 spans downstream (only right semi-span shown, Navier-Stokes propagation for second condition)

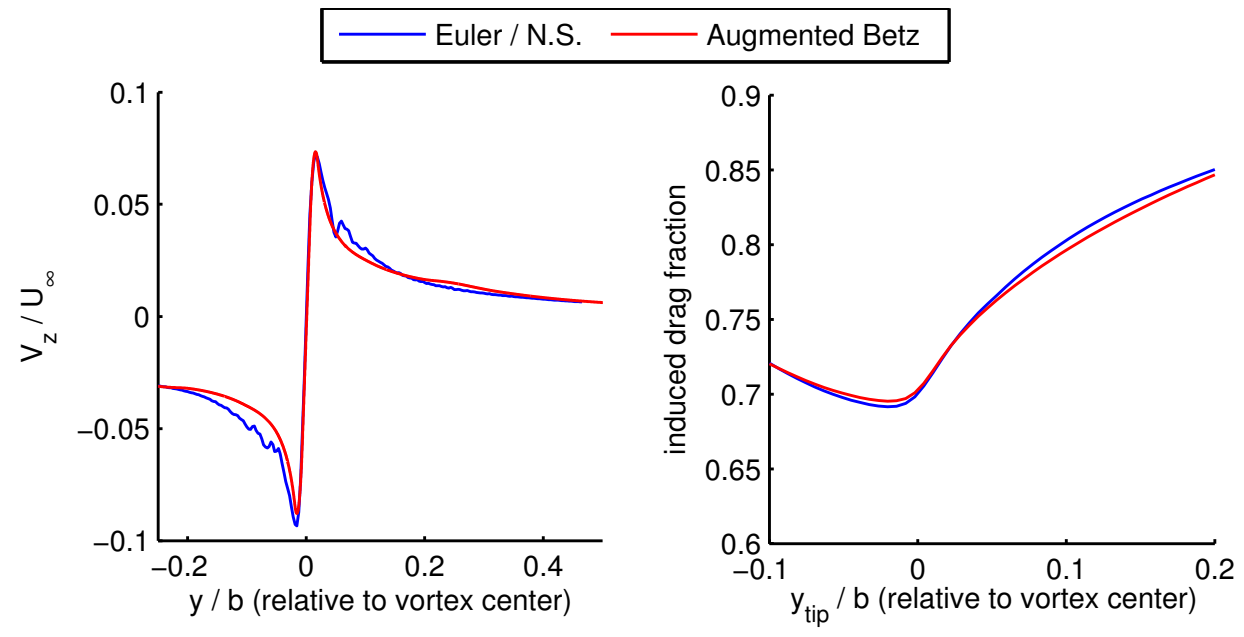

(c) Difference between high and low fidelity methods (20 spans downstream). Left figure shows upwash as a function of spanwise position on a cut through center of vortex. Right figure shows difference in estimated induced drag fraction for a trailing aircraft.

Figure 9. Wake development behind a transonic wing/body/nacelle $\left(M_{\infty}=0.785, C_{L}=0.52\right)$. 


\section{2-Aircraft Formations}

The methodology of the second approach is applied to formations of two identical transport aircraft separated streamwise by 20 wingspans. Variations in cruise Mach number, lateral positioning, and lift coefficient are examined.

\section{A. Geometries}

Two geometries are examined in this study. Both are transonic wing-body configurations whose airfoils use supercritical sections with blunt trailing edges. The first is based on the DLR-F4 geometry used in the 1st AIAA CFD Drag Prediction Workshop. ${ }^{22}$ Inviscid solutions of that geometry have been shown to significantly over-predict lift, resulting in a negative angle of attack for the aircraft at the design lift coefficient of $0.5 .^{23}$ In order to give a more realistic carry-through of lift across the fuselage, the wing was re-mounted on this geometry at a $4.5^{\circ}$ lower incidence angle so that the entire aircraft can fly at $2^{\circ}$ incidence at the design conditions. In addition, the aft end of the fuselage was extended to a point in order to avoid issues with properly converging the base drag of the fuselage. This increased the fineness ratio of the fuselage by about $5.6 \%$.

The second geometry is the wing and body components of the Common Research Model used in the 4th AIAA CFD Drag Prediction Workshop. ${ }^{24}$ A summary of the design conditions for the two geometries is seen in Table 1, and isometric views of the aircraft are seen in Figure 10. All force coefficients are based on the same reference areas used in the above mentioned references namely, $S_{r e f}=0.1454 \mathrm{~m}^{2}$ for Transport 1 and $S_{\text {ref }}=4,130 f t^{2}$ for Transport 2 .

Table 1. Design Conditions of the Two Representative Geometries

\begin{tabular}{cccccc}
\hline \hline & $C_{L}$ & $M_{\infty}$ & $R$ & $\Lambda_{Q C}$ & $\lambda$ \\
\hline Transport 1 & 0.5 & $\sim 0.75$ & 9.4 & $25^{\circ}$ & 0.3 \\
Transport 2 & 0.5 & $\sim 0.85$ & 9.0 & $35^{\circ}$ & 0.275 \\
\hline \hline
\end{tabular}

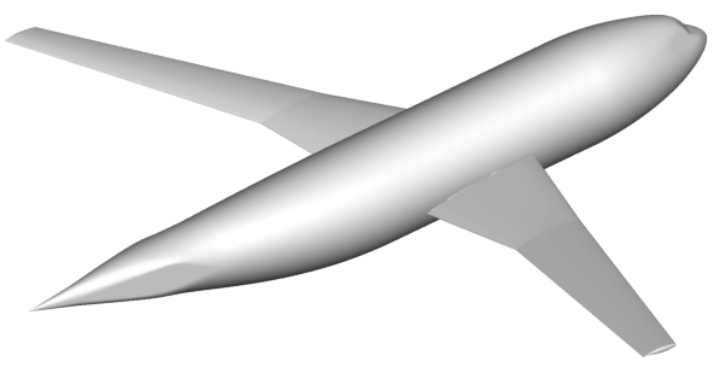

(a) Transport 1

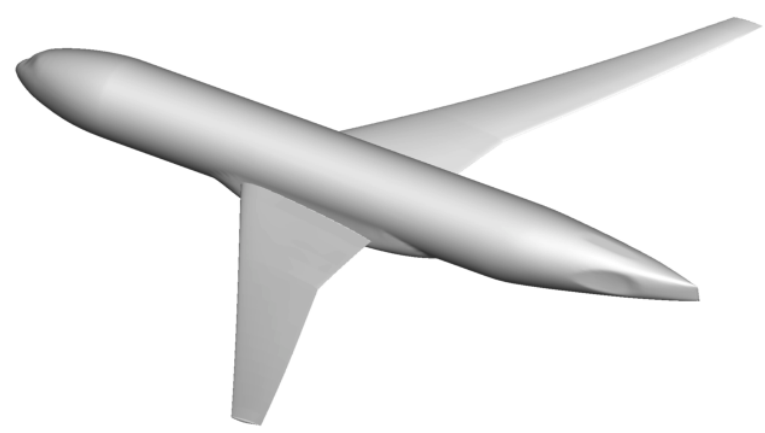

(b) Transport 2

Figure 10. Two transonic transport aircraft geometries. Both are wing/body configurations with supercritical sections.

No control surfaces are present on these geometries, so all results are for untrimmed configurations. Control surface deflection would add some trim drag, but trimming in roll would also provide some beneficial load alleviation on the more highly loaded sections close to the vortex. Thus, the predicted formation-induced compressibility penalties may be somewhat over-predicted in this analysis.

\section{B. Variation in Mach Number}

First, an estimate for the out-of-formation cruise speed for each aircraft is needed. The drag divergence Mach number can be estimated by examining the variation in the aircraft's lift-to-drag ratio as a function 
of Mach number. Since the minimum cost speed for commercial aircraft tends to be somewhat faster than the maximum range speed, the cruise speed is usually faster than the speed that maximizes the lift-to-drag ratio. A simple method to estimate the drag divergence Mach number is the Mach number at which the lift-to-drag ratio drops by about a couple percent from its maximum.

Since AERO package only computes the inviscid component of drag, an estimate of the viscous component is needed. This is done simply by assuming that near the cruise speed, parasite drag accounts for $55 \%$ of the total drag. This is a fairly typical value for transport aircraft in cruise. ${ }^{25}$ A small variation in parasite drag with Mach number is also included based on the decrease in turbulence skin friction coefficient at higher Mach numbers. ${ }^{\text {a }}$ This simple method is sufficient for our purposes because while the magnitude of the lift-to-drag ratio is sensitive to the particular choice of parameters, the general variation in performance with Mach number is insensitive across a range of reasonable parameters. The lift-to-drag ratio for the two transports is shown in Figure 11. Since cruise speeds are typically close to the drag divergence speed, in the following results it is assumed that Transport 1 has a cruise speed of about $M_{\infty}=0.76$, and Transport 2 has a cruise speed of about $M_{\infty}=0.83$.

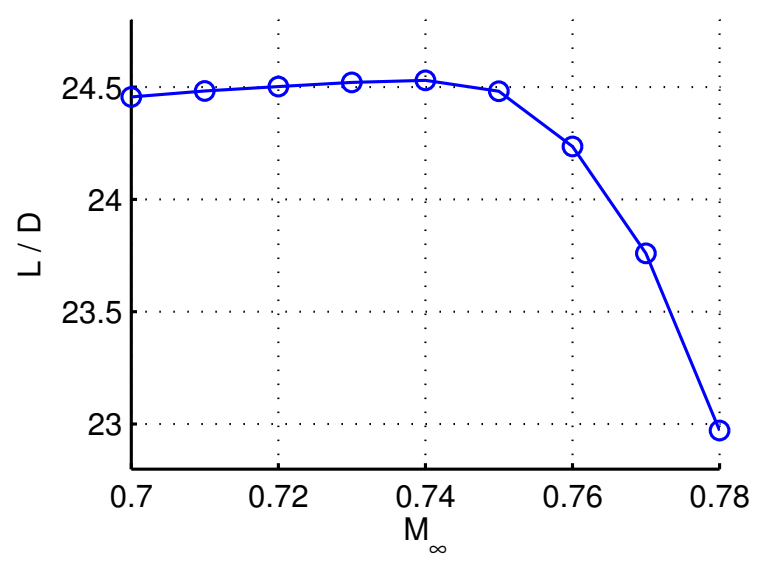

(a) Transport 1

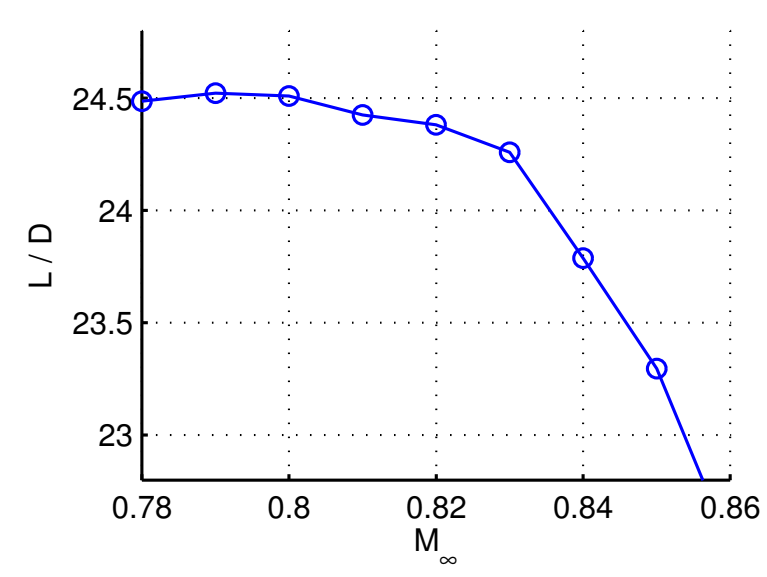

(b) Transport 2

Figure 11. Aircraft lift-to-drag ratio as a function of freestream Mach number (out-of-formation).

Predicting the optimal in-formation Mach number is not as straightforward. The inviscid drag for both the lead and trailing aircraft in a 2-aircraft extended formation is shown in Figure 12 for the two different transports. All cases are run at a constant lift coefficient of 0.5 for both lead and follower aircraft. While both aircraft see a similar trend with increased drag at higher Mach numbers, the drag rise is slightly more pronounced for the trailing aircraft.

Because the trends in the drag rise curves are similar, one might suggest that the optimal in-formation cruise speed should be essentially the same as the out-of-formation cruise speed. However, because the calculations are inviscid, a closer examination of the pressure distribution on the wing is needed. While for some conditions formation-induced shocks may not be large enough to manifest as large increases in compressibility drag, they could still be strong enough to separate the flow on the wing and make the aircraft un-flyable due to buffet. As a simple measure of shock strength, the maximum Mach number on the aft half of the local wing section is shown as a function of spanwise position in Figure 13 (Transport 2). The variation is shown for three different Mach numbers. Only the half of the wing nearest the incoming vortex is shown, as the other half of the wing is essentially unaffected by the vortex upwash. For reference, the out-of-formation cruise condition is also shown. For this analysis, any shocks stronger than the reference condition are deemed unacceptable. At the out-of-formation cruise speed, the trailing aircraft in-formation experiences significantly stronger shocks near the incoming vortex. The figure suggests that slowing down approximately $1.5 \%$ below the nominal drag divergence Mach number may be sufficient to alleviate the formation-induced compressibility penalties, though an examination of the pressure distributions suggests up to a $2.5 \%$ reduction in Mach number may be necessary.

\footnotetext{
${ }^{a}$ http://adg.stanford.edu/aa241/drag/skinfriction.html, accessed June 13, 2011
} 


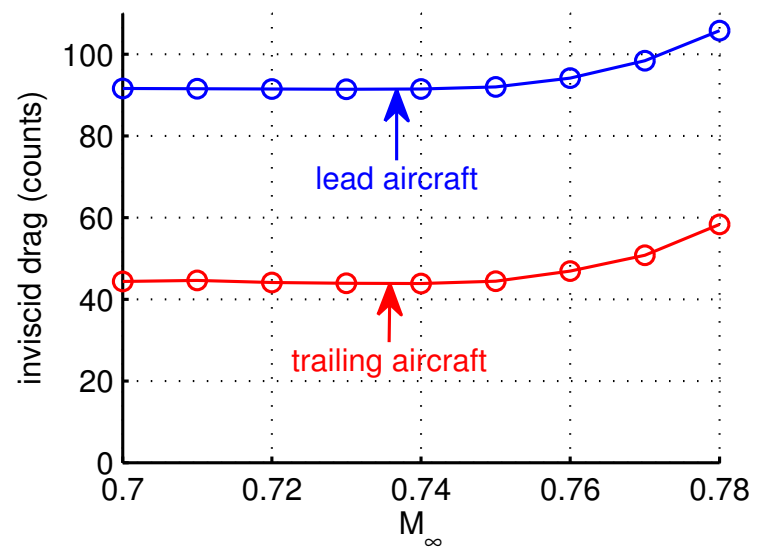

(a) Transport 1

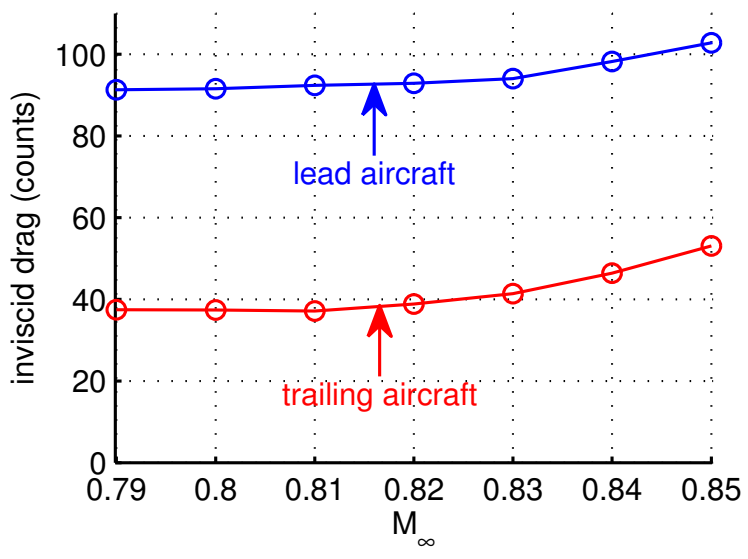

(b) Transport 2

Figure 12. Inviscid drag for lead and trailing aircraft is shown separately as a function of Mach number for a 2-aircraft homogeneous formation. Lift coefficient is held constant at $C_{L}=0.5$. For all in-formation cases the trailing aircraft's wing tip is positioned at the center of the incoming vortex.

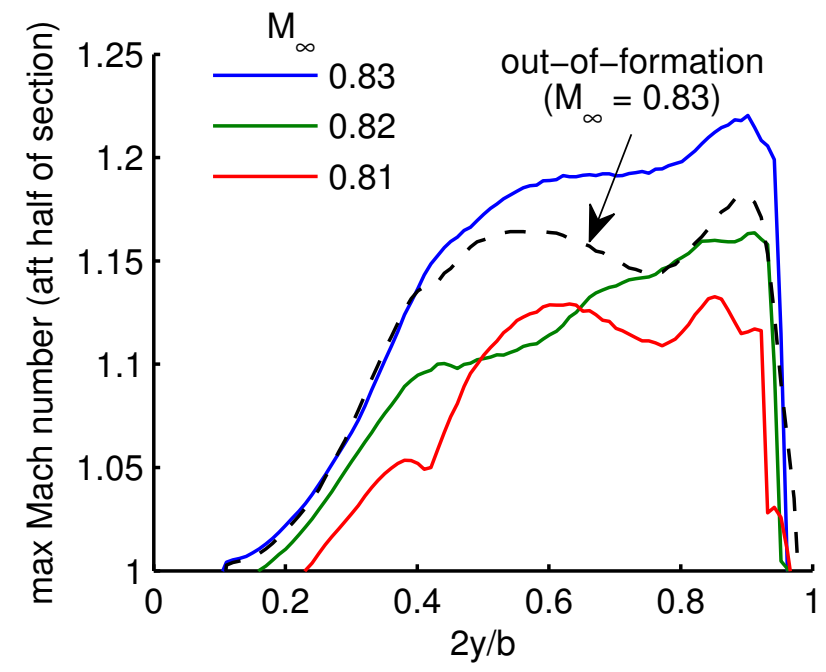

Figure 13. Maximum Mach number on the aft half of the local section as a function of spanwise position for Transport 2 (only half of wing nearest incoming vortex shown). In-formation cases are shown for three different Mach numbers, and the out-of-formation case in shown at the cruise condition. 
While that simple measure of shock strength provides some insight, the pressure distribution across the wing is examined in more detail at the conditions of interest. The $C_{p}$ distribution on the upper surface of the wing for the half of the wing nearest the vortex is shown in Figure 14 for Transport 1 and Figure 15 for Transport 2. The wing is shown at the cruise Mach number out-of-formation, the cruise Mach number in-formation, and at a reduced Mach number in-formation. $C_{p}$ cuts are also shown at a few stations along the wing. The reduced Mach number in the figures is approximately $2.5 \%$ below the out-of-formation dragdivergence Mach number.

The results suggest that by slowing down about $2-3 \%$ below the nominal drag divergence Mach number, the compressibility penalties of flying in-formation can be essentially eliminated. Shock strength is reduced to or below that of the wing at its out-for-formation cruise condition. The main difference in the formation cases is the stronger nose suction peak. This is generally acceptable, as the adverse pressure gradient on the back side of the peak should be tolerable near the nose of the airfoil where the boundary layer is still strong.

If the formation flies at a slower cruise speed, then the formation would also need to fly at a lower altitude or at a higher lift coefficient than the out-of-formation conditions (or some combination of both). A $2.5 \%$ reduction in Mach number corresponds roughly to a 1,000 ft drop in cruise altitude at fixed lift coefficient, or a $5 \%$ increase in lift coefficient at fixed altitude. A higher lift coefficient may be desirable, as incompressible analyses suggest that the optimal lift coefficient in-formation is higher then the out-of-formation optimum. ${ }^{6}$ However, this does not necessarily hold at transonic speeds and is explored further in a later section.

Finally, the Euler results are compared to the faster incompressible methodology used in our past work ${ }^{9}$ in Figure 16. The geometry used in the vortex-lattice model is re-twisted so that the out-of-formation lift distribution is reasonably well matched at the cruise condition. The figure shows the formation drag fraction, which is the total drag of all the aircraft in-formation relative to the drag of all aircraft out-of-formation at the cruise condition. Viscous drag is estimated using the simple method described previously. In plotting the formation drag fraction it is assumed that the magnitude of the viscous component of drag does not change when the aircraft are flown in formation. From the figure we see that the low-fidelity results agree well with the Euler solutions until compressibility effects start to become significant. 

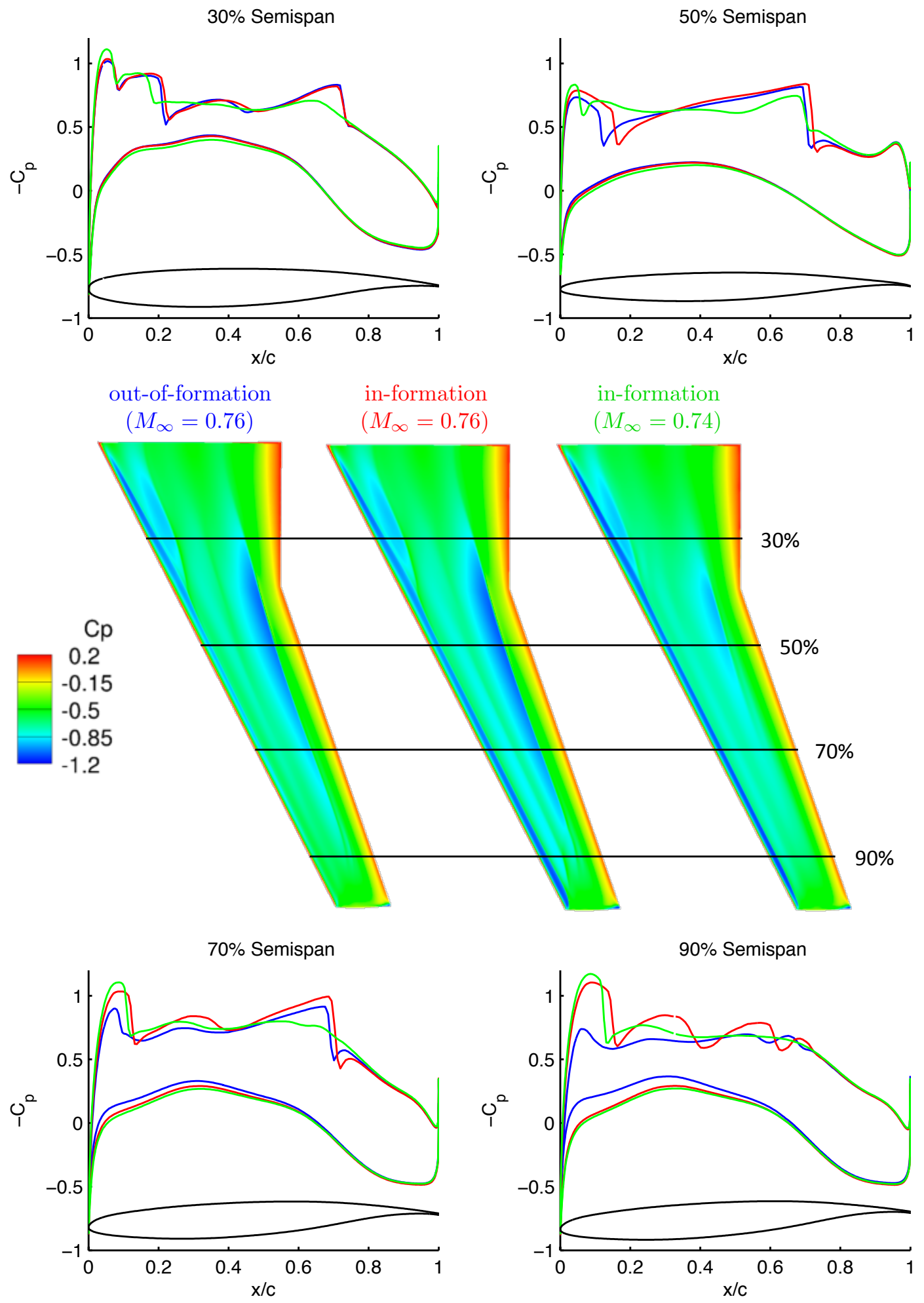

Figure 14. $C_{p}$ contours on upper surface of wing of Transport 1 at three conditions: out-of-formation at the cruise speed, in-formation at the cruise speed, and in-formation at a lower Mach number. For the in-formation cases, the aircraft is the trailing aircraft in a 2-aircraft formation, and the half of the wing shown is the one closest to the incoming vortex. The $C_{p}$ distribution is also shown at select cuts through the wing. 

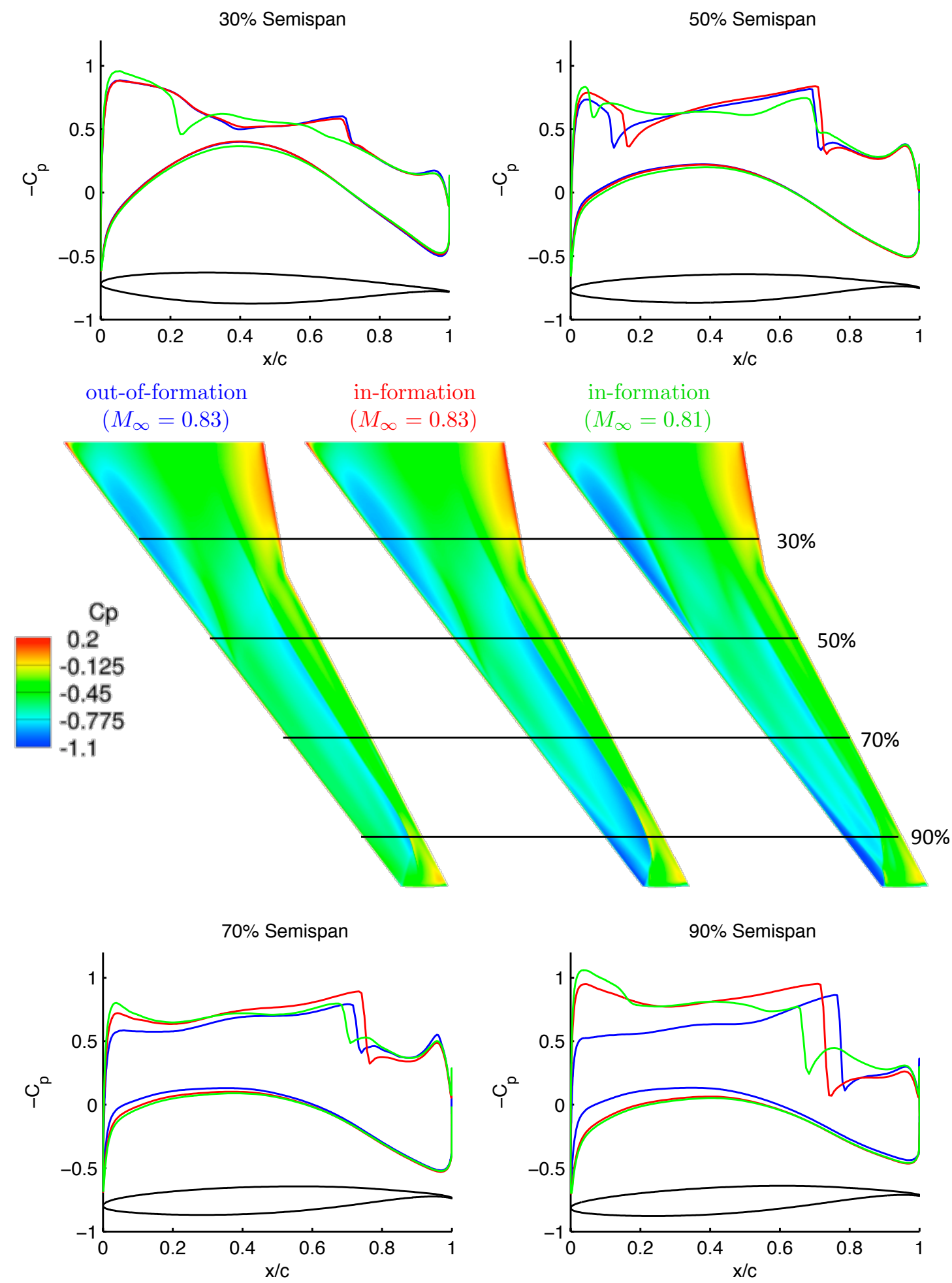

Figure 15. $C_{p}$ contours on upper surface of wing of Transport 2 at three conditions: out-of-formation at the cruise speed, in-formation at the cruise speed, and in-formation at a lower Mach number. For the in-formation cases, the aircraft is the trailing aircraft in a 2-aircraft formation, and the half of the wing shown is the one closest to the incoming vortex. The $C_{p}$ distribution is also shown at select cuts through the wing. 


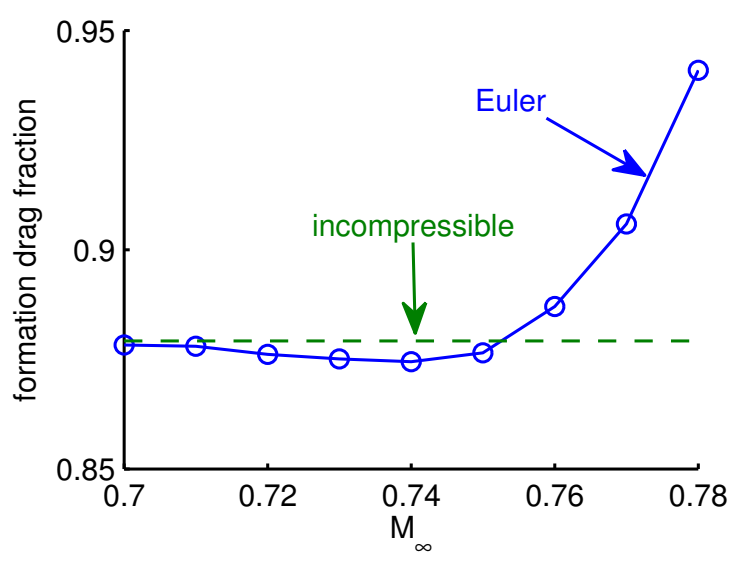

(a) Transport 1

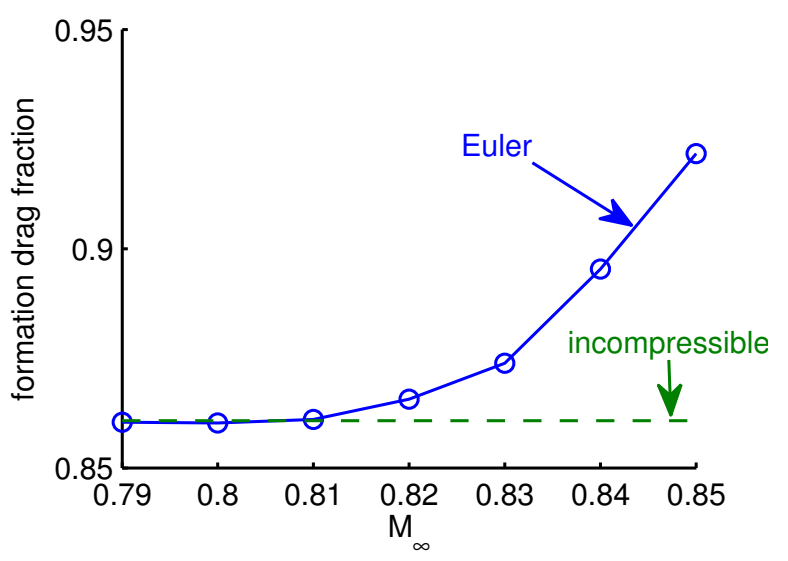

(b) Transport 2

Figure 16. Comparison of the formation drag fraction between an Euler solution and an incompressible aerodynamics analysis with far-field wake models.

\section{Variation in Lateral Positioning}

The motivation for this analysis is that while slowing down can reduce the formation-induced compressibility penalties, there are economic incentives to flying faster. By flying further from the vortex center some of the compressibility penalties may be reduced without having to slow down as much. The following study examines the effect of varying lateral separation, while keeping vertical position aligned with the center of the vortex. Vertical separation is expected to have a similar effect, although the distances would differ as formation drag savings are more sensitive to vertical separation. ${ }^{9}$ The definition for the relative spacing is seen in Figure 17. The variation in formation drag fraction with lateral spacing is shown in Figure 18 for three different Mach numbers, beginning with the cruise Mach number. A small reduction in Mach number shows a small benefit in terms of formation drag reduction, though further decreases seem to not make much of a difference. The incompressible low-fidelity estimates seem to follow the same trend as the Euler solutions.

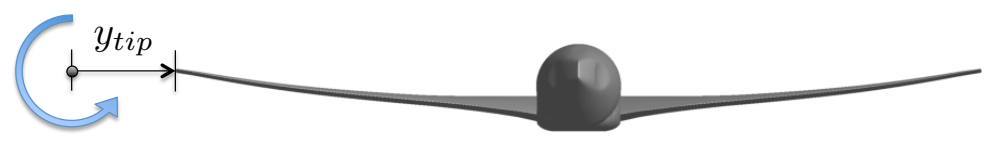

Figure 17. Positioning defined from center of nearest vortex from upstream aircraft to wingtip of trailing aircraft.

The compressibility penalty in-formation is examined using the simple metric of local Mach number on the aft half of the section in Figure 19 (Transport 2). The shock strength never quite returns to outformation levels, but becomes comparable when the trailing aircraft is 0.1 spans or more from the vortex center. However, at these separation distances the inviscid drag savings of the formation is reduced by about $10 \%$ or more. Most of the reduction in compressibility effects appears to occur for $y_{\text {tip }} / b<=0.05$. Larger spacings provide little additional benefit, especially considering the large loss in induced drag savings.

The pressure contours on the wing are shown for Transport 2 for three cases: flying out-of-formation, in-formation positioned right next to the vortex, and in-formation with $y_{\text {tip }} / b=0.05$ (Figure 20). All cases are at the cruise speed. While some alleviation in shock strength is seen from increasing separation distance from the vortex, at $y_{\text {tip }} / b=0.05$ it is not enough without slowing down as well. A $1-2 \%$ reduction in Mach number combined with $y_{t i p} / b=0.05$ eliminates the formation-induced compressibility penalty for this formation. More details for Transport 1 are not shown as the conclusions are similar. Optimal positioning and cruise speed would need to evaluated in the context of a specific mission where the relative costs of fuel consumption and flight time can be assessed. 


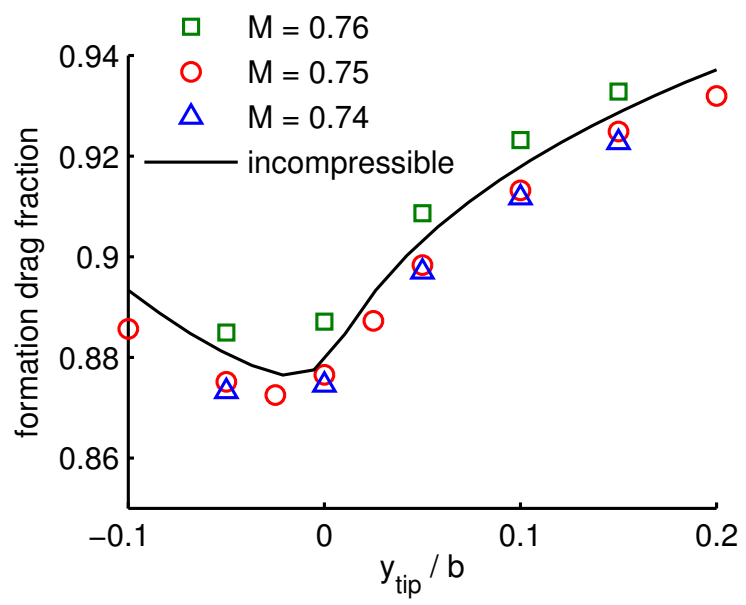

(a) Transport 1

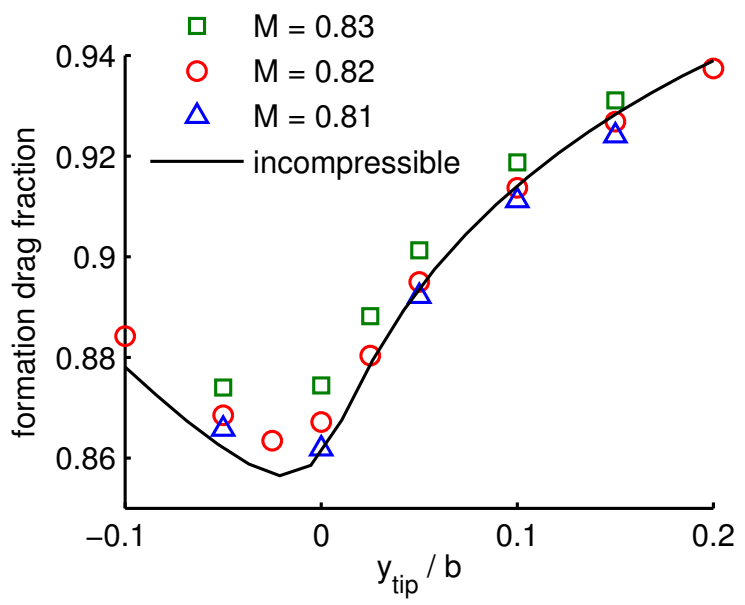

(b) Transport 2

Figure 18. Formation drag fraction of the formation as a function of the position of the trailing aircraft's wingtip relative to the incoming vortex center. Results are shown for three different Mach numbers, beginning with the cruise Mach number. Also shown for comparison is a low-fidelity incompressible estimate.

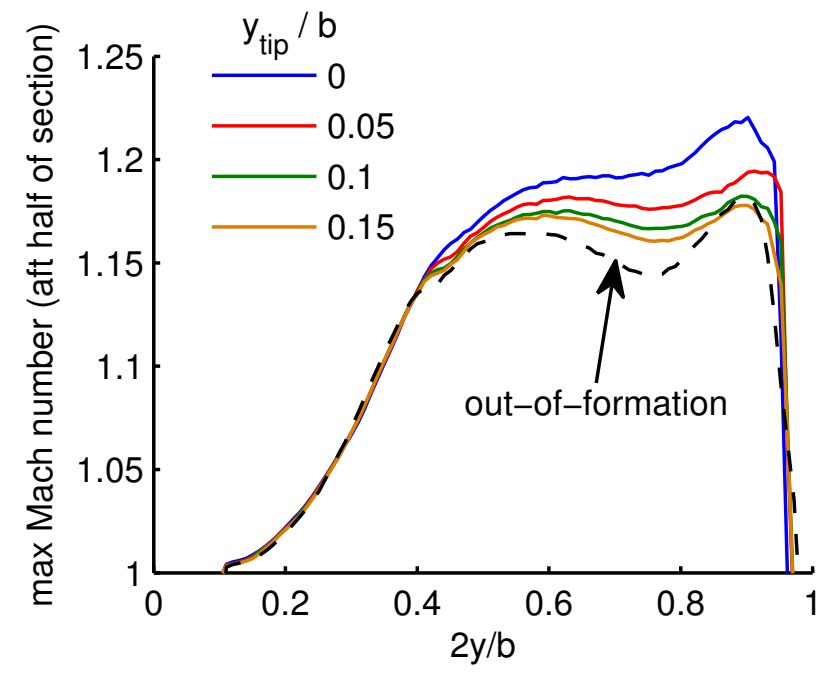

Figure 19. Maximum Mach number on aft half of the local section as a function of spanwise position for Transport 2 (only half of wing nearest incoming vortex shown). In-formation cases are shown for four different lateral spacings, and the out-of-formation condition is shown for reference. 

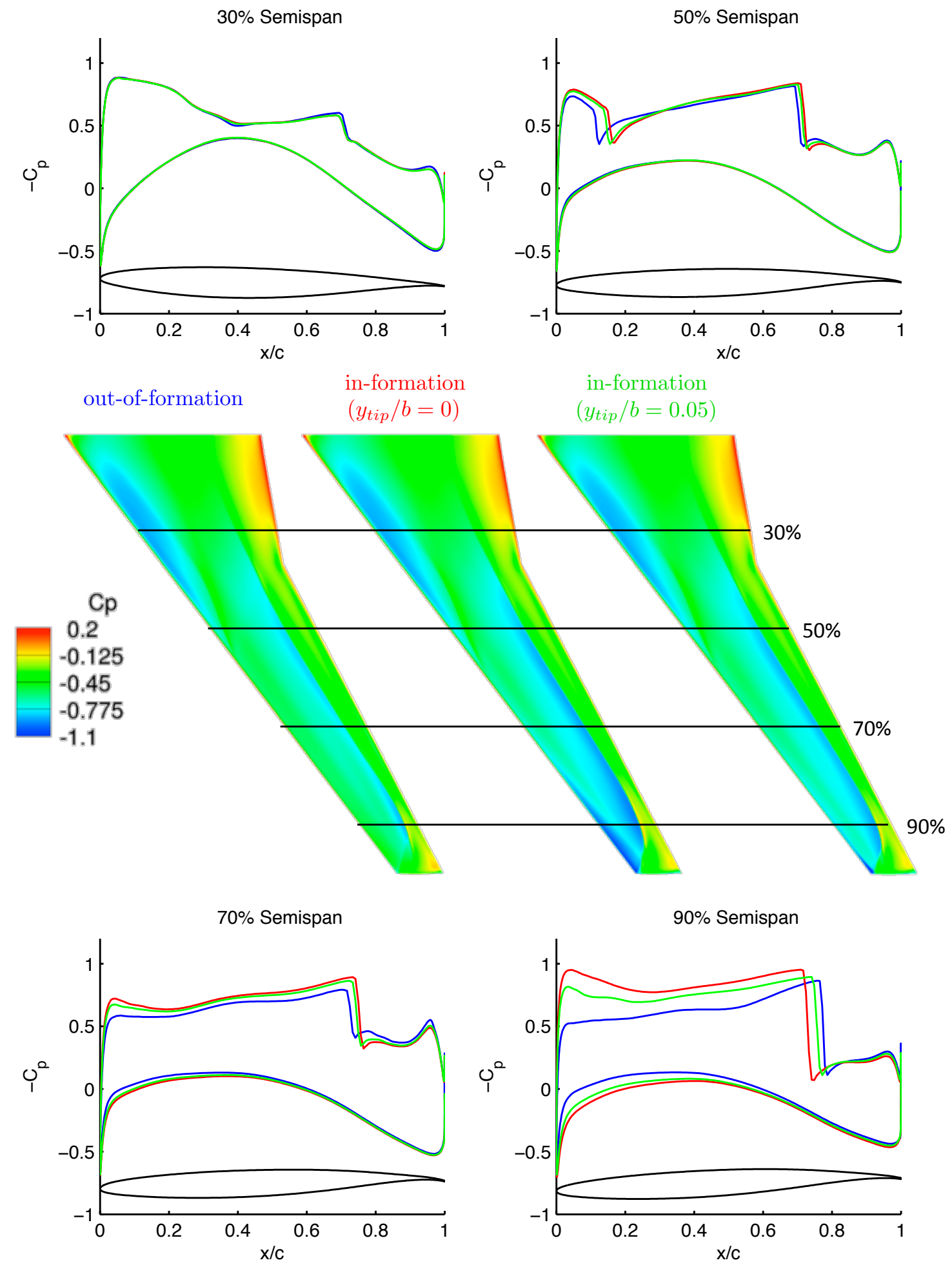

Figure 20. $C_{p}$ contours on upper surface of wing of Transport 2 at three conditions all at the cruise speed: out-of-formation, in-formation with wingtip aligned with vortex center, and in-formation with $y_{\text {tip }} / b=0.05$. For the in-formation cases, the aircraft is the trailing aircraft in a 2-aircraft formation, and the half of the wing shown is the one closest to the incoming vortex. 


\section{Variation in Lift Coefficient}

Incompressible analyses suggest that the optimal formation lift coefficient is higher than the solo lift coefficient. However, the effects of compressibility will decrease the optimal formation lift coefficient at cruise speeds. Because this analysis depends on the tradeoff in induced versus all other forms of drag, an estimate for the viscous component of drag is needed. Parasite drag is estimated simply as discussed previously. Additionally, a small viscous increment is added as a function of lift coefficient:

$$
C_{D i \text { viscous }}=K C_{D p} C_{L}^{2}
$$

where $\mathrm{K}$ is based on flight tests of commercial transports. ${ }^{26}$ While the specific values of lift-to-drag ratio, and the optimal lift coefficient will vary with the value of the assumed parameters, the general conclusions of this section remain the same for reasonable variation in these parameters.

Figure 21 shows the variation in formation drag fraction as a function of lift coefficient for a few different Mach numbers. The incompressible estimate is also shown. We see, as expected, that as the freestream Mach number is increased, the optimal lift coefficient is decreased. Still, moderate increases in lift coefficient may be realizable, and can yield a small performance benefit. We have already seen that flying slower can eliminate the compressibility penalties of flying in-formation. By simultaneously flying at a higher $C_{L}$ the formation can have a small increase in performance, and maintain design altitude. Figure 22 compares the pressure distribution for Transport 2 at its out-of-formation cruise condition, and in-formation at a reduced Mach number but at fixed altitude.

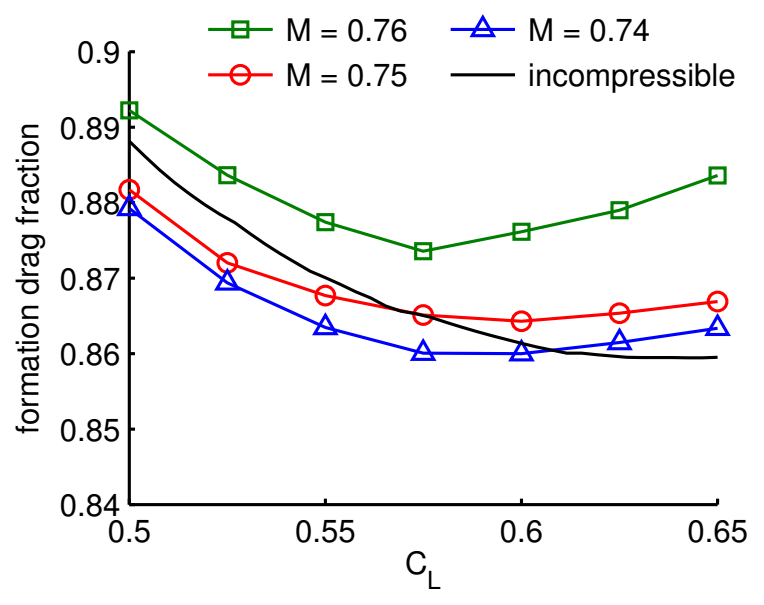

(a) Transport 1

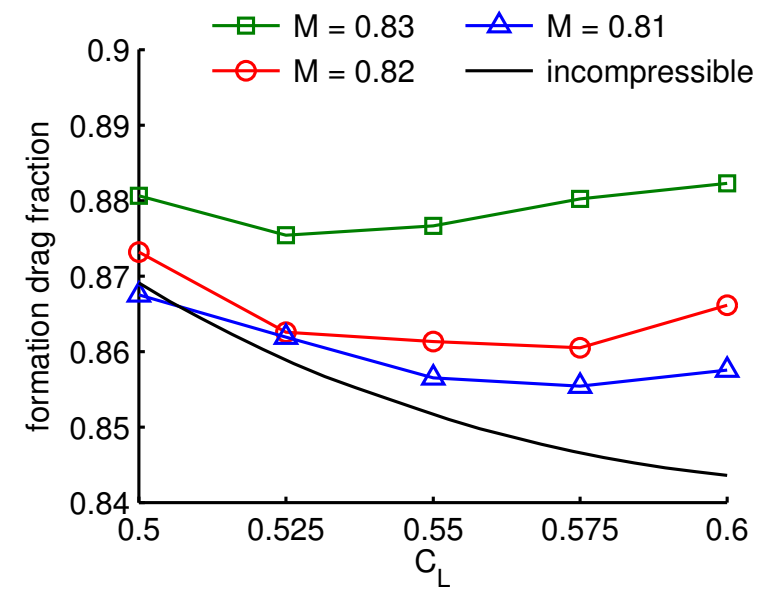

(b) Transport 2

Figure 21. Variation in formation drag fraction as a function of lift coefficient. As expected, formation flight has improved aerodynamic performance at higher lift coefficients, but not as high as predicted by incompressible analyses. 

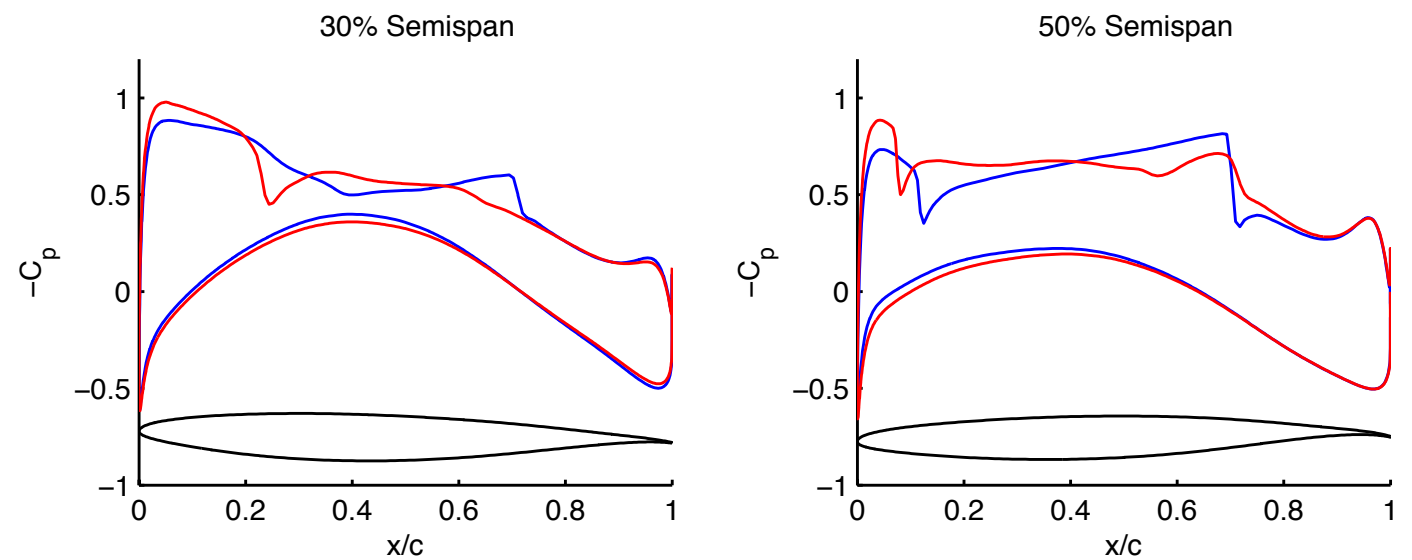

out-of-formation

in-formation

$\left(M_{\infty}=0.83, C_{L}=0.5\right) \quad\left(M_{\infty}=0.81, C_{L}=0.525\right)$

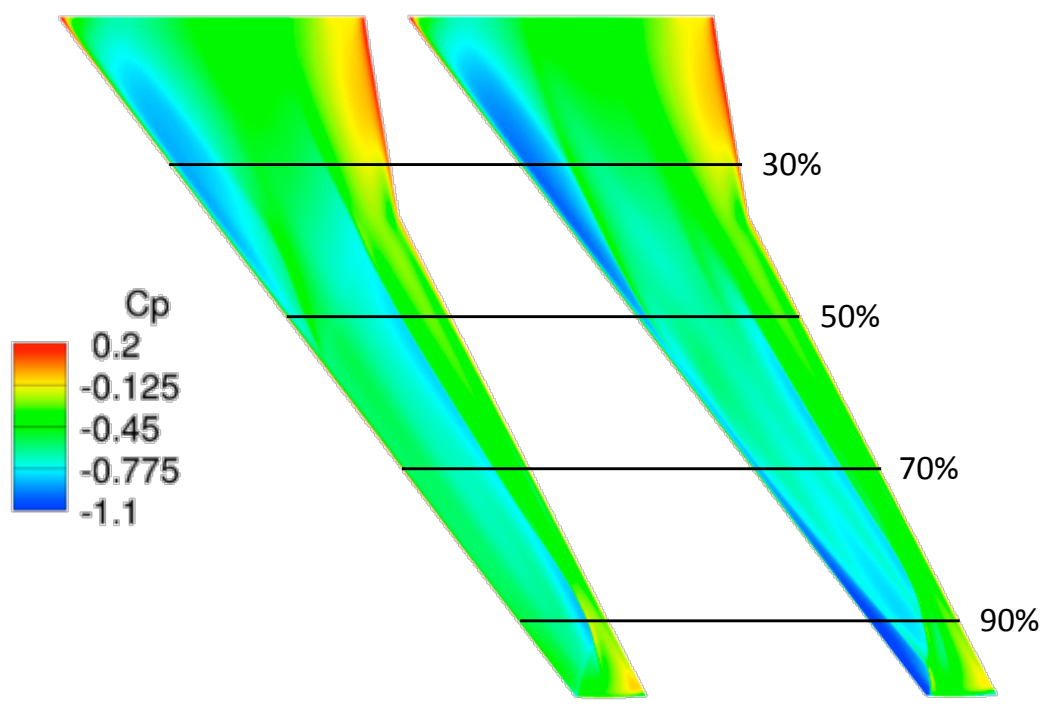

$70 \%$ Semispan

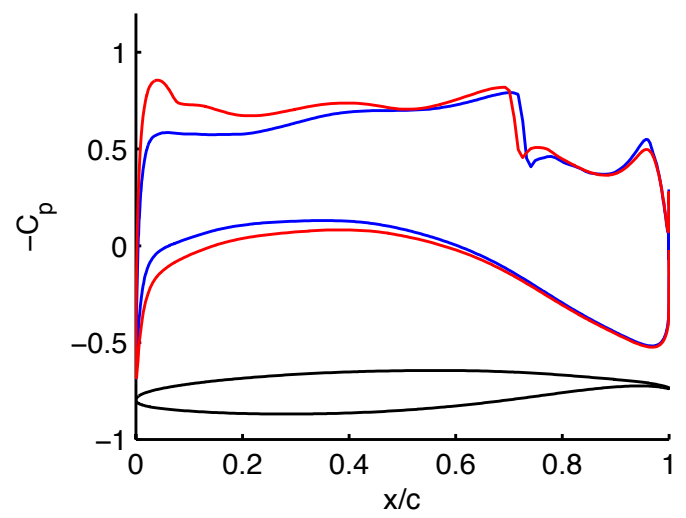

$90 \%$ Semispan

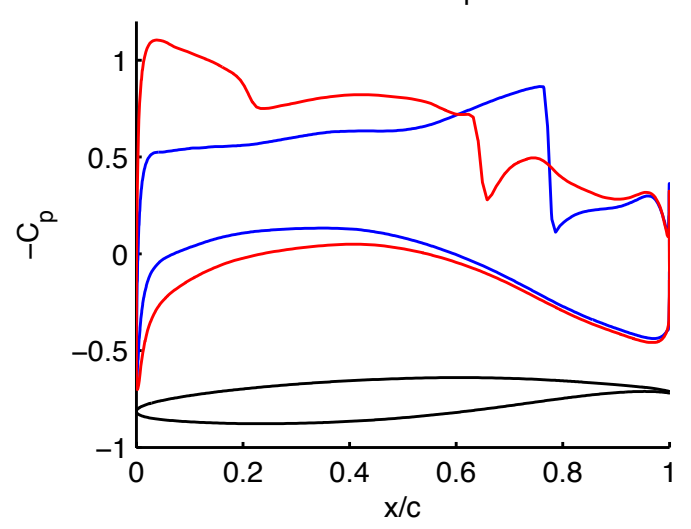

Figure 22. Comparison between Transport 2 at its out-of-formation cruise condition, and in a 2-aircraft formation with a reduced Mach number but fixed altitude. 


\section{Conclusion}

We have examined two methods for propagating a wake downstream of an aircraft for distances in the range of approximately 10-50 wingspans. The far-field conservation-of-vorticity method, combined with empirical data agrees well with the 2D Navier-Stokes solutions for the configurations considered here. This method is computationally efficient and provides an efficient boundary condition for any level of mesh refinement. Formations with deflected control surfaces, or with more than two aircraft, may require the more detailed CFD approach.

The results for homogeneous 2-aircraft formations of transonic wing/body aircraft have shown that formation flight is not efficient at the out-of-formation drag divergence Mach number. At these conditions, the formation-induced shocks may not necessarily imply large compressibility drag increases in inviscid solutions, but they do show strong potential for flow separation and buffet. However, slowing down by 2-3\% in Mach number essentially eliminates the formation-induced compressibility penalties (for identical aircraft, positioned to fly very close to the vortex). This reduction in speed would require the formation to fly about $1,000 \mathrm{ft}$ lower than the normal cruise altitude, or fly at a $5 \%$ higher lift coefficient. This latter option has the additional benefit that the total drag of the formation decreases for modest increases in lift coefficient. Slowing down is an intriguing option in that a reduction in cruise Mach number of even a few percent from todays speeds has the potential for significant reductions in environmental impacts, ${ }^{27}$ as well as synergistic savings with other next generation technologies such as natural laminar flow wings and open rotor engines. The disadvantage to slowing down is the increased flight time (about 10 minutes on an 8 hour flight) and associated higher costs.

Formations with larger lateral separation between aircraft permit slightly higher in-formation cruise Mach number (about 1-2\% below nominal drag divergence with $5 \%$ span spacing). Of course, the increased offset distance is accompanied by a $5 \%$ increase in induced drag. There seems to be little incentive to flying any further from the vortex as the reduction in compressibility effects becomes disproportionately smaller, and induced drag savings decrease significantly.

While these results provide some insight into the compressibility effects of extended formation flight, more exploration is needed. Studies that could yield further understanding include studying the effect of aircraft trim in-formation, incorporating more detailed geometries, using viscous solvers, investigating formations with more than two aircraft and with heterogeneous aircraft, designing multi-point optimal wings, conducting more experiments and flight tests, and performing full mission analyses to further evaluate the tradeoffs in cruise speed, aircraft positioning, and cruise altitude. Some of these studies are currently underway.

\section{Acknowledgments}

The authors gratefully acknowledge the contributions of Michael Aftosmis, Marian Nemec, and James Kless of NASA Ames Research Center. Many aspects of the methodology have benefitted from their expertise and suggestions. Michael and Marian are developers of the AERO package and in addition to general support in its use, they have modified the code to support the vortex boundary condition used in this work. The Euler simulations in this study were done using computational resources provided by NASA Ames. This work is part of a broader research program on formation flight at Stanford University kindly supported by Airbus UK.

\section{References}

\footnotetext{
${ }^{1}$ Boeing Commercial Airplanes, Current Market Outlook 2010-2029, 2010.

${ }^{2}$ Airbus, Global Market Forecast 2009-2028, 2009.

${ }^{3}$ Wieselsberger, C., "Beitrag zur Erklärung des Winkelfluges einiger Zugvögel," Z. Flugtechnik 8 S Motorluftschiffahrt, Vol. 5, 1914, pp. 225-229.

${ }^{4}$ Lissaman, P. B. S. and Shollenberger, C. A., "Formation Flight of Birds," Science, Vol. 168, No. 3934, 1970, pp. 1003, doi:10.1126/science.168.3934.1003.

${ }^{5}$ Hummel, D., "Aerodynamic Aspects of Formation Flight in Birds," Journal of Theoretical Biology, Vol. 104, No. 3, 1983, pp. 321-347.

${ }^{6}$ Blake, W. and Multhopp, D., "Design, Performance and Modeling Considerations for Close Formation Flight," AIAA Atmospheric Flight Mechanics Conference and Exhibit, AIAA-1998-4343, August 1998, pp. 476-486.

${ }^{7}$ Wagner, E., Jacque, D., Blake, W., and Pachter, M., "Flight Test Results of Close Formation Flight for Fuel Savings," AIAA Atmospheric Flight Mechanics Conference and Exhibit, August 2002.
} 
${ }^{8}$ Ray, R. J., Cobleigh, B. R., Vachon, M. J., and John, C. S., "Flight Test Techniques Used to Evaluate Performance Benefits During Formation Flight," TP-2002-210730, NASA, August 2002.

${ }^{9}$ Ning, S. A., Flanzer, T., and Kroo, I., "Aerodynamic Performance of Extended Formation Flight," Journal of Aircraft, Vol. 48, No. 3, May 2011, pp. 855-865, doi:10.2514/1.54636.

${ }^{10}$ Gerz, T. and Holzäpfel, F., "Wing-Tip Vortices, Turbulence, and the Distribution of Emissions," AIAA Journal, Vol. 37, No. 10, October 1999, pp. 1270-1276, doi:10.2514/2.595.

${ }^{11}$ Widnall, S. E., "The Structure and Dynamics of Vortex Filaments," Annual Review of Fluid Mechanics, Vol. 7, No. 1, 1975, pp. 141-165, doi:10.1146/annurev.fl.07.010175.001041.

${ }^{12}$ Aftosmis, M. and Berger, M., "Multilevel Error Estimation and Adaptive h-Refinement for Cartesian Meshes with Embedded Boundaries," AIAA Aerospace Sciences Meeting, AIAA 2002-0863, January 2002.

${ }^{13}$ Nemec, M. and Aftosmis, M. J., "Adjoint Error Estimation and Adaptive Refinement for Embedded-Boundary Cartesian Meshes," AIAA Computational Fluid Dynamics Conference, AIAA 2007-4187, June 2007.

${ }^{14}$ Nemec, M. and Aftosmis, M. J., "Adjoint Sensitivity Computations for an Embedded-Boundary Cartesian Mesh Method," Journal of Computational Physics, Vol. 227, No. 4, 2008, pp. 2724-2742, doi:10.1016/j.jcp.2007.11.018.

${ }^{15}$ Rennich, S. and Lele, S., "Numerical Method for Incompressible Vortical Flows with Two Unbounded Directions," Journal of Computational Physics, Vol. 137, No. 1, Oct 1997, pp. 101-129, doi:10.1006/jcph.1997.5796.

${ }^{16}$ Betz, A., "Behavior of Vortex Systems," TM-713, NACA, 1933.

${ }^{17}$ Delisi, D., Greene, G., Robins, R., Vicroy, D., and Wang, F., "Aircraft Wake Vortex Core Size Measurements," AIAA Applied Aerodynamics Conference, AIAA-2003-3811, June 2003.

${ }^{18}$ Holzäpfel, F., "Probabilistic Two-Phase Wake Vortex Decay and Transport Model," Journal of Aircraft, Vol. 40, No. 2, March 2003, pp. 323-331, doi:10.2514/2.3096.

${ }^{19}$ Wintzer, M., "Span Efficiency Prediction Using Adjoint-Drive Mesh Refinement," Journal of Aircraft, Vol. 47, No. 4, July 2010, pp. 1468-1470, doi:10.2514/1.C031049.

${ }^{20}$ Donaldson, C. d., Snedeker, R. S., and Sullivan, R. D., "Calculation of Aircraft Wake Velocity Profiles and Comparison With Experimental Measurements," Journal of Aircraft, Vol. 11, No. 9, September 1974, pp. 547-555, doi:10.2514/3.60385.

${ }^{21}$ Rossow, V. J., "On the Inviscid Rolled-Up Structure of Lift-Generated Vortices," Journal of Aircraft, Vol. 10, No. 11, 1973, pp. 647-650, doi:10.2514/3.60277.

${ }^{22}$ Redeker, G., "A Selection of Experimental Test Cases for the Validation of CFD Codes," AGARD AR, Vol. 2, No. 303, August 1994

${ }^{23}$ Aftosmis, M. J., Berger, M. J., and Alonso, J. J., "Applications of a Cartesian Mesh Boundary-Layer Approach for Complex Configurations," AIAA Aerospace Sciences Meeting, AIAA-2006-0652, January 2006.

${ }^{24}$ Vassberg, J. C., DeHaan, M. A., Rivers, S. M., and Wahls, R. A., "Development of a Common Research Model for Applied CFD Validation Studies," AIAA Applied Aerodynamics Conference, AIAA 2008-6919, August 2008.

${ }^{25}$ Leoviriyakit, K. and Jameson, A., "Multi-point Wing Planform Optimization via Control Theory," AIAA Aerospace Sciences Meeting, AIAA 2005-0450, January 2005.

${ }^{26}$ Shevell, R., Fundamentals of Flight, Prentice Hall, 2nd ed., 1989.

${ }^{27}$ Dallara, E. S. and Kroo, I. M., "Aircraft Design for Reduced Climate Impact," AIAA Aerospace Sciences Meeting, AIAA 2011-265, January 2011. 